\title{
Low-frequency radio observations of Seyfert galaxies: A test of the unification scheme ${ }^{\star}$
}

\author{
V. Singh ${ }^{1,2,3}$, P. Shastri ${ }^{2}$, C. H. Ishwara-Chandra ${ }^{3}$, and R. Athreya ${ }^{3,4}$ \\ ${ }^{1}$ Institut d'Astrophysique Spatiale, Bât. 121, Université Paris-Sud, 91405 Orsay Cedex, France \\ e-mail: veeresh.singh@ias.u-psud.fr \\ 2 Indian Institute of Astrophysics, 560034 Bangalore, India \\ 3 National Center for Radio Astrophysics - TIFR, 411007 Pune, India \\ ${ }^{4}$ Indian Institute of Science Education and Research, 411008 Pune, India
}

Received 24 December 2012 / Accepted 28 March 2013

\begin{abstract}
Aims. We present low-frequency radio imaging and spectral properties of a well-defined sample of Seyfert galaxies using GMRT 240/610 MHz dual frequency observations. Radio spectra of Seyfert galaxies over $240 \mathrm{MHz}$ to $5.0 \mathrm{GHz}$ are investigated using $240 \mathrm{MHz}, 610 \mathrm{MHz}$ flux densities derived from GMRT, and $1.4 \mathrm{GHz}$ and 5.0 GHz flux densities mainly from published VLA data. We test the predictions of Seyfert unification scheme by comparing the radio properties of Seyfert type 1s and type $2 \mathrm{~s}$.

Methods. We chose a sample such that the two Seyferts subtypes have matched distributions in parameters that are independent of the orientation of AGN, obscuring torus, and the host galaxy. Our sample selection criteria allowed us to assume that the two Seyfert subtypes are intrinsically similar within the framework of the unification scheme.

Results. The new observations at 240/610 MHz, together with archival observations at $1.4 \mathrm{GHz}, 5.0 \mathrm{GHz}$ show that types $1 \mathrm{~s}$ and $2 \mathrm{~s}$ have statistically similar radio luminosity distributions at $240 \mathrm{MHz}, 610 \mathrm{MHz}, 1.4 \mathrm{GHz}$, and $5.0 \mathrm{GHz}$. The spectral indices at selected frequency intervals $\left(\alpha_{240 \mathrm{MHz}}^{610 \mathrm{MHz}}, \alpha_{610 \mathrm{MHz}}^{1.4 \mathrm{GH}}\right.$, and $\left.\alpha_{1.4 \mathrm{GHz}}^{5.0 \mathrm{GHz}}\right)$, as well as index measured over $240 \mathrm{MHz}$ to $5.0 \mathrm{GHz}\left(\alpha_{\mathrm{int}}\right)$ for the two Seyfert subtypes, have similar distributions with median spectral index $(\alpha) \sim-0.7\left(S_{v} \propto \gamma^{\alpha}\right)$, consistent with the synchrotron emission from optically thin plasma. In our snapshot 240/610 MHz GMRT observations, most of the Seyfert galaxies primarily show an unresolved central radio component, except for a few sources in which faint kpc-scale extended emission is apparent at $610 \mathrm{MHz}$. Our results on the statistical comparison of the multifrequency radio properties of our sample Seyfert galaxies agree with the predictions of the Seyfert unification scheme.
\end{abstract}

Key words. galaxies: Seyfert - galaxies: active - radio continuum: galaxies

\section{Introduction}

Seyfert galaxies are categorized as low-luminosity $\left(M_{B \text {-Band }}>\right.$ -23; Schmidt \& Green 1983), radio-quiet $\left(\frac{F_{5.0 \mathrm{GHz}}}{F_{B-\mathrm{Band}}}<10\right.$; Kellermann et al. 1989) active galactic nuclei (AGN), hosted in spiral or lenticular galaxies (Weedman 1977). Depending on the presence or absence of the broad permitted emission lines in their nuclear optical spectra, Seyfert galaxies are classified as "type 1" and "type 2", respectively. The detection of broad permitted emission lines in the spectropolarimetric observations of few Seyfert type 2s laid the foundation for the Seyfert unification scheme (Antonucci \& Miller 1985). The unification scheme hypothesizes that Seyfert type $1 \mathrm{~s}$ and type $2 \mathrm{~s}$ constitute the same parent population and appear different solely because of the differing orientations of the dusty molecular obscuring material having a torus-like geometry around the AGN. In Seyfert type $2 \mathrm{~s}$, the dusty torus intercepts the observer's lineof-sight and blocks the direct view of the broad line region and accreting black hole. While, in type $1 \mathrm{~s}$, the observer's line-ofsight is away from the obscuring torus, and thus, broad line region and accreting black hole are directly visible (Antonucci \& Miller 1985; Antonucci 1993; Urry et al. 1995).

^ Figures 2, 4 and Appendix A are available in electronic form at http://www . aanda.org
There have been various studies yielding both consistent and inconsistent results to the validity of the Seyfert unification scheme. Some of the key results that support the unification scheme include, the presence of broad emission lines in the polarized optical and infrared spectra of many Seyfert $2 \mathrm{~s}$ (Antonucci \& Miller 1985; Moran et al. 2000), the biconical structure of the narrow line region (Mulchaey et al. 1996), the similar amount of total molecular gas detected by CO measurements in the two Seyfert subtypes (Maiolino et al. 1997), the systematic higher X-ray absorption in type 2s (Cappi et al. 2006; Singh et al. 2011), and similar nuclear radio properties of both the subtypes (Lal et al. 2011). However, results inconsistent to the unification scheme remain, such as the absence of hidden Seyfert 1 nuclei in several Seyfert 2s (Tran 2001, 2003), higher tendency for Seyfert 1s to be hosted in galaxies of earlier Hubble type (Malkan et al. 1998), the lack of X-ray absorption in some Seyfert 2s (Panessa \& Bassani 2002), and the higher propensity of nuclear starbursts in Seyfert 2s (Buchanan et al. 2006).

It has been argued that the sample selection is the most crucial issue in testing the predictions of the Seyfert unification scheme, and the samples used in many previous studies suffer from subtle biases (Antonucci 2002). There are suggestions that the optical and UV selected samples are likely to have inherent biases against obscured sources (Ho \& Ulvestad 2001). Infrared selected samples can be biased towards unusually dusty sources, 
as well as towards sources with a higher level of nuclear star formation (Ho \& Ulvestad 2001; Buchanan et al. 2006). X-ray selected samples from flux-limited surveys are likely to have obscured type 2 Seyferts that are intrinsically more luminous than the selected type 1 counterparts (Heckman et al. 2005; Wang et al. 2009).

Recent studies on testing the unification scheme have emphasized and attempted to use less-biased samples and reported results that are consistent with the scheme (Cappi et al. 2006; Dadina 2008; Beckmann et al. 2009; Gallimore et al. 2010; Lal et al. 2011). Nonetheless, issues related to sample selection remain, and the quest to test the validity and limitations of the Seyfert unification with more improved and well-defined samples continues. Keeping these sample-selection arguments in mind, we attempt to test the predictions of unification scheme by using a Seyfert sample in which types $1 \mathrm{~s}$ and $2 \mathrm{~s}$ have matched distributions in parameters that are independent of the orientation of the obscuring torus and AGN axis. Our selection criteria mitigate the biases that are generally inherent in samples derived from flux-limited surveys, and it also allows us to assume that the two Seyfert subtypes are not intrinsically different within the framework of the unification scheme (Schmitt et al. 2003a; Lal et al. 2011).

In this paper we attempt to investigate the low-frequency radio properties of the two Seyfert subtypes to test the predictions of the unification scheme. High-resolution radio observations show that Seyfert nuclei produce weak bipolar radio-emitting jets that are largely confined within the host galaxy (Thean et al. 2000; Lal et al. 2004). However, at low frequencies, significant radio emission may also arise from the extended emission related either to nuclear activity (Colbert et al. 1996; Gallimore et al. 2006) or to the star-formation (Baum et al. 1993). Notably, most of the Seyfert samples have been studied at higher frequencies $(\geq 1.4 \mathrm{GHz})$ with high-resolution ( $\sim \operatorname{arcsec}$ or less) observations (Ulvestad \& Wilson 1984a,b, 1989; Kukula et al. 1995; Morganti et al. 1999; Nagar et al. 1999; Thean et al. 2000, 2001; Lal et al. 2011), which effectively filter out emission from lowsurface-brightness and extended radio structures. In this paper we study the low-frequency radio emission properties of Seyfert galaxies using the Giant Meterwave Radio Telescope (GMRT) dual-frequency 240/610 MHz observations. Hitherto, there has been dearth of low-frequency $(<1.0 \mathrm{GHz})$ radio observations of the sample of Seyfert galaxies, and our observations are the first attempt, to our knowledge, to systematically study the lowfrequency radio properties of a well-defined sample of Seyfert galaxies. To investigate the nature of multifrequency radio spectra, we utilize $1.4 \mathrm{GHz}, 5.0 \mathrm{GHz}$ flux density measurements derived mainly from Very Large Array (VLA) "D" configuration observations that are sensitive to the low-surface-brightness radio emission.

This paper is structured as follows. Our sample and its selection criteria are described in Sect. 2. The details of our observations and data reductions are described in Sect. 3. The comparison of radio properties of Seyfert type 1s and type $2 \mathrm{~s}$ are discussed in Sect. 4. Radio properties of individual sources are given in Appendix A. Wherever required, we assume cosmological parameters $H_{0}=71 \mathrm{~km}^{-1} \mathrm{Mpc}^{-1}, \Omega_{\mathrm{m}}=0.27$, and $\Omega_{\mathrm{vac}}=0.73$.

\section{The sample}

Our sample consists of 20 Seyfert galaxies with 10 type 1 s and 10 type $2 \mathrm{~s}$. All the sample sources satisfy the basic criteria of being Seyfert galaxy i.e., (i) sources are radio-quiet AGN
$\left(\frac{F_{5.0 \mathrm{GHz}}}{F_{B-\text { band }}}<10\right)($ Kellermann et al. 1989); (ii) AGN's optical $B$-band absolute magnitude $\left(M_{B}\right)$ is less than -23 (Schmidt \& Green 1983); (iii) the nuclear line width (FWHM) of the permitted line $\mathrm{H}_{\beta}$ is larger than $1000 \mathrm{~km} \mathrm{~s}^{-1}$ for Seyfert type 1s (Khachikian \& Weedman 1974); (iv) the line intensity ratio of [O III] $\lambda 5007 \AA$ to $\mathrm{H}_{\beta}$ is greater that 3.0 for Seyfert type $2 \mathrm{~s}$ (Dahari \& De Robertis 1988); and (v) sources are hosted in spiral or lenticular galaxies (Weedman 1977). Thus, we ensure that our Seyfert sample is not contaminated by LINERs, quasars, and radio-loud AGNs. Also, our sample sources fall into the "Seyfert region" of [O III]/O[ II] versus $[\mathrm{O}$ I $] / \mathrm{H}_{\alpha}$ diagnostic diagram of Kewley et al. (2006). Seyfert galaxies that show any broad permitted emission line component in their optical spectra (i.e., subclasses 1.0, 1.2, 1.5, 1.8, 1.9) are considered as type 1, while those which show only narrow permitted emission lines are considered as type 2 .

Our sample selection criteria are based on the method proposed by Lal et al. (2011). An elaborate discussion on the sample selection issues and adopted methodology is presented in Lal et al. (2011). We chose our sample using isotropic properties that are independent of the orientation of the obscuring torus, AGN, and host galaxy. The criterion of using isotropic orientationindependent parameter mitigates biases that are caused by obscuring torus or by the orientation of the AGN jet-axis or host galaxy. It is to be noted that the inherent biases in Seyfert samples selected from optical, UV, and X-ray surveys are interpreted due to the obscuration mainly from the torus (Ho \& Ulvestad 2001; Heckman et al. 2005). The criterion of matching the distributions of the two subtypes in the orientation-independent parameters allows us to assume that two Seyfert subtypes in our sample are intrinsically similar within the framework of the unification scheme. Essentially, we ascertain that we are not comparing intrinsically different sources. We consider five orientation-independent parameters i.e., cosmological redshift, [O III] $\lambda 5007 \AA$ luminosity, Hubble type of the host galaxy, total absolute stellar luminosity of the host galaxy, and absolute bulge magnitude. These parameters are independent of the orientation of obscuring torus, AGN, or the host galaxy and are also intimately linked to the evolution of AGN and host galaxy. A brief description of the chosen orientation-independent parameters is given below.

(i) Cosmological redshift: it allows us to have control over the cosmological evolution effect. In our sample the two Seyfert subtypes have similar distributions of cosmological redshift spanning a narrow interval. This implies that the two Seyfert subtypes in our sample belong to similar cosmological epochs.

(ii) [O III] $\lambda 5007 \AA$ line luminosity: it originates in the narrow line region that is outside the torus and therefore, it is not affected by the torus obscuration. Also, [O III] $\lambda 5007 \AA$ luminosity is expected to be correlated with nuclear ionizing continuum, as well as nuclear X-ray luminosity, and can be considered as the proxy for intrinsic AGN power (Nelson \& Whittle 1995; Heckman et al. 2005). In our sample the two subtypes are chosen such that they have similar narrow distributions of [O III] luminosity. This allows us to assume that the AGN powers of the two subtypes are matched.

(iii) Hubble type of the host galaxy: it considers the effect of the host galaxy's morphology and its environment on AGN evolution and vice-versa. The Hubble type of a galaxy does not depend on the orientation of the torus or AGN-jet axis (Pringle et al. 1999). In our sample two Seyfert subtypes have similar distributions of Hubble type, although 
matching is less strong for Hubble stage $(\mathrm{T})$ values higher than 2. The values of Hubble stage for our sample sources are taken from Malkan et al. (1998) and de Vaucouleurs et al. (1991).

(iv) Total stellar absolute magnitude of the host galaxy: it can be considered as a characteristic property of the host galaxy. The total stellar absolute magnitudes of the host galaxies of our sample sources are taken from (Whittle 1992), and these are corrected for non-stellar and emission line flux, redshift $(K)$ correction, the internal absorption, and the Galactic absorption.

(v) Absolute magnitude of the bulge: it is independent to the orientation of the obscuring torus, AGN axis. Also, the absolute magnitude of the bulge is roughly proportional to the mass of the central supermassive black hole (Kormendy \& Gebhardt 2001; McConnell \& Ma 2013), arguably a fundamental parameter of the AGN system. The values of absolute magnitude of the bulge in our sample sources are taken from Whittle (1992).

We chose our sample of 20 Seyfert galaxies (10 type 1s and 10 type 2 s) such that the two subtypes have matched distributions in all five orientation-independent parameters. Our sample is extracted from the Whittle (1992) sample of 140 Seyfert galaxies (78 type $1 \mathrm{~s}$ and 62 type $2 \mathrm{~s}$ ). To ensure the bona fide nature of Seyfert type, we selected only those sources that host in spiral or lenticular galaxies i.e., Hubble type SO or later (Weedman 1977). After excluding sources with early type, peculiar, or uncertain host galaxy morphologies, we obtained a sample of 92 Seyferts (47 type $1 \mathrm{~s}$ and 45 type 2 s). To minimize the effects of obscuration by the host galaxy disk on the optical properties, we selected Seyferts with relatively face-on host galaxies (i.e., ratio of minor to major isophotal diameter axis greater than 0.5 ), noting that there is no correlation between AGN and the host galaxy's axis (Pringle et al. 1999; Nagar \& Wilson 1999). It resulted in a sample of 76 (41 type 1s and 35 type $2 \mathrm{~s}$ ) Seyfert galaxies. Of these 76 sources, 7 (2 type $1 \mathrm{~s}$ and 5 type $2 \mathrm{~s}$ ) sources had declination (Dec $\leq-53^{\circ}$ ) beyond the GMRT coverage, and were excluded. In the sample of 69 (39 type $1 \mathrm{~s}$ and 30 type $2 \mathrm{~s}$ ) sources, we further imposed the redshift cutoff $z \geq 0.031$, as per our selection criteria of choosing sources from narrow a span of cosmological redshift. The redshift cut off rendered the sample of 49 (23 type 1 s and 26 type 2 s) sources. To ensure that the source is easily detected above $6 \sigma$ in a ten-minute snapshot scan with GMRT, we picked sources that had $1.4 \mathrm{GHz} \mathrm{NVSS}^{1} /$ FIRST $^{2}$ flux density higher than $6.0 \mathrm{mJy}$ i.e., corresponding to an extrapolated $610 \mathrm{MHz}$ flux density assuming a flatter radio spectral index of -0.5 , higher than $9.0 \mathrm{mJy}$. This yielded a sample of 41 (19 type $1 \mathrm{~s}$ and 22 type $2 \mathrm{~s}$ ) Seyfert galaxies. From these 41 Seyferts we picked 20 Seyferts (10 type $1 \mathrm{~s}$ and 10 type $2 \mathrm{~s}$ ) that satisfied our selection criteria i.e., matched distributions of the two subtypes in the orientationindependent parameters and could be observed in our limited telescope observing time. All our sample sources comply with the observing feasibility with GMRT, i.e., sources are within the GMRT declination coverage range and are detectable with sufficient signal-to-noise ratio in a ten-minute snapshot with GMRT. There is no strong radio source in the neighborhood that can affect the dynamical range, i.e., flux density of target source.

Table 1 lists our sample sources and the values of their orientation-independent parameters. Redshift values are taken from the NASA Extragalactic Database (NED) and are rounded

\footnotetext{
http://www.cv.nrao.edu/nvss/

2 http://sundog.stsci.edu/index.html
}

off to the fourth decimal place. The values of [O III] luminosity, absolute stellar magnitude, and absolute bulge magnitude are taken from Whittle (1992). A quality factor has been assigned to all the parameters in Whittle (1992) and it reflects the level of reliability. We have used only those parameter values that have a reliable quality rating, i.e., "a" to "c" in Whittle (1992) catalog. We opted for updated values of parameters whenever available in the literature; for example, [O III] luminosity and Hubble stage values obtained from HST observations (Schmitt et al. 2003a; Malkan et al. 1998) were preferred over values given in Whittle (1992). HST observations of [O III] emission reported in Schmitt et al. (2003a) yield the spatially resolved [O III] emission associated with NLR regions. A comparison between [O III] fluxes obtained from HST and ground-based observations shows that both kinds of observations give similar [O III] flux measurements (Fig. 2 in Schmitt et al. 2003a), and therefore, in general, contamination to [O III] emission by HII regions is likely not to be significant in Seyfert galaxies. The presence of circumnuclear starburst may affect the measurements of [O III] flux, bulge, and stellar magnitudes. However, most of our sample sources are free of circumnuclear starburst contamination, except NGC 3227 and NGC 7469, which are known to possess circumnuclear starburst (Gonzalez Delgado \& Perez 1997; Genzel et al. 1995). Thus, our sample selection is not very affected by the circmnuclear starburst contamination.

Figure 1 shows the matched distributions of the orientationindependent parameters for the two Seyfert subtypes of our sample. The matched distributions allow us to assume that we are not comparing entirely intrinsically different sources selected from different parts of the (luminosity, bulge mass, Hubble type, redshift) evolution function (Schmitt et al. 2003b). Indeed, following the same sample selection criteria, there is a possibility of obtaining a larger sample; however, we would like to emphasize that the sample selection is more important and not the sample size to rigorously test the predictions of the unification scheme. Larger but heterogeneous and biased sample is likely to lead to incorrect conclusions. The relatively small size of our sample is the result of the combined effect of restrictive selection criteria and observational constraints. And, in future, we plan to extend our analysis to a larger sample.

\section{Observations and data reduction}

We carried out full-array GMRT (Swarup et al. 1991), snapshot observations at dual-frequency $610 / 240 \mathrm{MHz}$ using four second integration time and a bandwidth of $32 \mathrm{MHz}$. Our observing $\log$ is given in Table 2. All the 20 Seyfert galaxies are observed with two to four scans (except NGC 7469 with only one scan and NGC 5548 with five scans) with each scan spanning to ten minutes. We gave more observing scans to weaker radio sources and less to stronger ones by using $1.4 \mathrm{GHz}$ NVSS flux densities as the indicator of source radio strength. Absolute flux and bandpass calibration is done by observing standard flux calibrators 3C 147 and 3C 286, at the start and end of the observing run. The phase calibration is done by observing a nearby phase calibrator source for nearly four minutes, before and after each scan of the target source. Our data are reduced in a standard way using "Astronomical Image Processing System" $(\mathrm{AIPS})^{3}$ package. For each run, bad visibility points are edited out, after which the data were calibrated. The edited and calibrated visibilities were Fourier-transformed into radio maps using "IMAGR" task in AIPS. We performed wide field imaging

\footnotetext{
http://www.aips.nrao.edu
} 
Table 1. Our Seyfert sample.

\begin{tabular}{|c|c|c|c|c|c|c|c|}
\hline $\begin{array}{l}\text { Source } \\
\text { name }\end{array}$ & $\begin{array}{c}\mathrm{RA} \\
(\mathrm{h} \mathrm{m} \mathrm{s})\end{array}$ & $\begin{array}{c}\text { Dec } \\
(\mathrm{d} \mathrm{m} \mathrm{s})\end{array}$ & $\begin{array}{c}\text { Redshift } \\
(z)\end{array}$ & $\begin{array}{c}\left.\log L_{[\mathrm{O}}^{\mathrm{III}]}\right] \\
\left(\mathrm{erg} \mathrm{s}^{-1}\right)\end{array}$ & $\begin{array}{c}\text { Hubble } \\
\text { stage }(\mathrm{T})\end{array}$ & $M_{B_{(\text {Total })}}$ & $M_{B_{(\text {Bulge })}}$ \\
\hline \multicolumn{8}{|c|}{ Sevfert $1 \mathrm{~s}$} \\
\hline MRK 6 & 065212.2 & +742537 & $0.0188^{7}$ & $41.79^{2}$ & $0^{5}$ & $-20.30^{1}$ & $-19.44^{1}$ \\
\hline NGC 3227 & 102330.6 & +195154 & $0.0039^{7}$ & $40.31^{1}$ & $1^{6}$ & $-21.47^{1}$ & $-20.46^{1}$ \\
\hline NGC 3516 & 110647.5 & +723407 & $0.0088^{7}$ & $41.07^{2}$ & $0^{5}$ & $-21.61^{1}$ & $-20.88^{1}$ \\
\hline NGC 4151 & 121032.6 & +392421 & $0.0033^{7}$ & $41.35^{1}$ & $2^{6}$ & $-21.22^{1}$ & $-19.98^{1}$ \\
\hline MRK 766 & 121826.5 & +294846 & $0.0129^{7}$ & $41.61^{2}$ & $3^{5}$ & $-21.03^{1}$ & $-20.10^{1}$ \\
\hline MRK 279 & 135303.4 & +691830 & $0.0305^{7}$ & $41.46^{1}$ & $1^{5}$ & $-21.59^{1}$ & $-20.98^{1}$ \\
\hline NGC 5548 & 141759.5 & +250812 & $0.0172^{7}$ & $41.42^{2}$ & $1^{5}$ & $-21.82^{1}$ & $-20.89^{1}$ \\
\hline ARK 564 & 224239.3 & +294331 & $0.0247^{7}$ & $41.38^{1}$ & $1^{6}$ & $-21.65^{1}$ & $-20.11^{1}$ \\
\hline NGC 7469 & 230315.6 & +085226 & $0.0163^{7}$ & $41.51^{1}$ & $4^{5}$ & $-22.01^{1}$ & $-20.90^{1}$ \\
\hline \multicolumn{8}{|c|}{ Seyfert $2 s$} \\
\hline MRK 348 & 004847.1 & +315725 & $0.0150^{7}$ & $41.31^{2}$ & $0^{5}$ & $-21.13^{1}$ & $-20.20^{1}$ \\
\hline MRK 1 & 011607.2 & +330522 & $0.0159^{7}$ & $41.52^{1}$ & $5^{5}$ & $-20.32^{1}$ & $-19.46^{1}$ \\
\hline MRK 1066 & 025958.6 & +364914 & $0.0120^{7}$ & $40.88^{1}$ & $5^{5}$ & $-21.06^{1}$ & $-20.45^{1}$ \\
\hline NGC 2110 & 055211.4 & -072722 & $0.0078^{7}$ & $40.35^{1}$ & $1^{5}$ & $-21.57^{1}$ & $-20.72^{1}$ \\
\hline NGC 2273 & 065008.6 & +605045 & $0.0061^{7}$ & $40.09^{3}$ & $1^{6}$ & $-20.99^{1}$ & $-19.97^{1}$ \\
\hline NGC 5252 & 133815.9 & +043233 & $0.0230^{7}$ & $41.41^{4}$ & $0^{5}$ & $-21.96^{1}$ & $-21.35^{1}$ \\
\hline NGC 5728 & 144223.9 & -171511 & $0.0094^{7}$ & $41.11^{1}$ & $1^{6}$ & $-22.35^{1}$ & $-21.12^{1}$ \\
\hline NGC 7212 & 220701.3 & +101352 & $0.0266^{7}$ & $42.15^{2}$ & $1^{6}$ & $-21.24^{1}$ & $-20.22^{1}$ \\
\hline NGC 7682 & 232903.9 & +033200 & $0.0171^{7}$ & $41.16^{1}$ & $0^{5}$ & $-21.11^{1}$ & $-19.88^{1}$ \\
\hline MRK 533 & 232756.7 & +084645 & $0.0289^{7}$ & $41.99^{2}$ & $5^{5}$ & $-22.65^{1}$ & $-20.69^{1}$ \\
\hline
\end{tabular}

Notes. Column 1: source name; Cols. 2 and 3: right ascension (hours, minutes, and seconds) and declination (degrees, arcminutes, and arcseconds) in J2000; Col. 4: cosmological redshift; Col. 5: [O III] $\lambda 5007$ Å luminosity in log; Col. 6: Hubble stage (T); Col. 7: total stellar absolute magnitude $\left(M_{B_{\text {Total }}}\right)$ corrected for the nuclear non-stellar continuum and emission line flux, redshift correction $(\mathrm{K})$, the internal absorption and the Galactic absorption; Column 8: absolute bulge magnitude in " $B$ " band $\left(M_{B_{\text {Bulge }}}\right)$.

References. (1) Whittle (1992); (2) Schmitt et al. (2003a); (3) Ferruit et al. (2000); (4) Polletta et al. (1996); (5) Malkan et al. (1998); (6) de Vaucouleurs et al. (1991); (7) NASA Extragalactic Database (NED).

as the primary beam size of full array GMRT at $610 / 240 \mathrm{MHz}$ is rather large $\sim 43^{\prime} / 114^{\prime}$. For all our sample sources, the signalto-noise ratio is high enough to apply self-calibration, which removes antenna-based phase and amplitude errors.

\section{Radio properties of Seyfert galaxies}

From our GMRT observations we obtained $240 \mathrm{MHz}$ and $610 \mathrm{MHz}$ radio images of all 20 Seyfert galaxies of our sample (cf. Fig. 2, Tables 3 and 4). In the following sections we discuss the radio properties (i.e., luminosities, spectra, and morphologies) of Seyfert types 1s and $2 \mathrm{~s}$ in the framework of the Seyfert unification scheme.

\subsection{Radio luminosities of Seyfert types $1 \mathrm{~s}$ and $2 \mathrm{~s}$}

The obscuring torus around the AGN is optically thin at centimeter radio wavelengths and there is no relativistic beaming effect in Seyfert galaxies (Shastri et al. 2003; Middelberg et al. 2004; Ulvestad et al. 2005). Therefore, the measured radio luminosity is expected to be independent of the orientation of the obscuring torus and the radio jet axis. According to the unification scheme, both the Seyfert subtypes are intrinsically similar, and therefore both types $1 \mathrm{~s}$ and $2 \mathrm{~s}$ are expected to show similar radio luminosities. This prediction of the unification scheme has been tested in some previous studies that yield varying results. For example, early studies reported in de Bruyn \& Wilson (1978) and Ulvestad \& Wilson (1984a) suggest that Seyfert type 2s are more radio powerful than Seyfert type 1s at centimeter wavelengths, giving results that are inconsistent with the unification scheme. However, later studies (Ho \& Ulvestad 2001) have argued that the samples used in earlier studies were biased towards radio-powerful Seyfert type $2 \mathrm{~s}$. High-resolution $3.6 \mathrm{~cm}$ VLA observations have shown that the two Seyfert subtypes have similar radio luminosities in samples based on $12 \mu \mathrm{m}$ (Thean et al. 2001) and $60 \mu \mathrm{m}$ (Schmitt et al. 2001). We made a statistical comparison of the radio luminosities of the two subtypes using $240 \mathrm{MHz}$, $610 \mathrm{MHz}$ GMRT observations and $1.4 \mathrm{GHz}$ (NVSS), 5.0 GHz observations of relatively low resolution $\left(\sim 20^{\prime \prime}-45^{\prime \prime}\right)$. Our observations are likely to pick up extended kpc-scale emission along with the nuclear AGN emission. In the unification scheme, both Seyfert subtypes expected to have similar likelihood of the presence of kpc-scale radio emission. Therefore, types 1s and $2 \mathrm{~s}$ are expected to show similar luminosity distributions obtained with low, as well as, high frequency/resolution radio observations.

Figure 3 shows the radio luminosity distributions at $240 \mathrm{MHz}, 610 \mathrm{MHz}, 1.4 \mathrm{GHz}$, and $5.0 \mathrm{GHz}$ of the type $1 \mathrm{~s}$ and type $2 \mathrm{~s}$ of our sample Seyferts. Seyfert type $1 \mathrm{~s}$ have $240 \mathrm{MHz}, 610 \mathrm{MHz}, 1.4 \mathrm{GHz}$, and $5.0 \mathrm{GHz}$ luminosities in the range of $L_{240 \mathrm{MHz}} \sim 6.09 \times 10^{28}-7.87 \times 10^{30} \mathrm{erg} \mathrm{s}^{-1} \mathrm{~Hz}^{-1}$, $L_{610 \mathrm{MHz}} \sim 5.30 \times 10^{28}-4.58 \times 10^{30} \mathrm{erg} \mathrm{s}^{-1} \mathrm{~Hz}^{-1}, L_{1.4 \mathrm{GHz}} \sim$ $3.49 \times 10^{28}-2.24 \times 10^{30} \mathrm{erg} \mathrm{s}^{-1} \mathrm{~Hz}^{-1}$, and $L_{5.0 \mathrm{GHz}} \sim 1.25 \times$ $10^{28}-8.37 \times 10^{29} \mathrm{erg} \mathrm{s}^{-1} \mathrm{~Hz}^{-1}$, respectively, with the me-

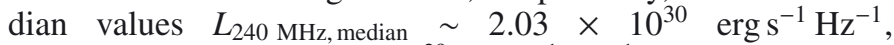
$L_{610 \mathrm{MHz}, \text { median }} \sim 7.88 \times 10^{29} \mathrm{erg} \mathrm{s}^{-1} \mathrm{~Hz}^{-1}, L_{1.4 \mathrm{GHz}}$, median $\sim$ $4.16 \times 10^{29} \mathrm{erg} \mathrm{s}^{-1} \mathrm{~Hz}^{-1}$, and $L_{5.0 \mathrm{GHz}}$, median $\sim 1.56 \times$ $10^{29} \mathrm{erg} \mathrm{s}^{-1} \mathrm{~Hz}^{-1}$, respectively. While type $2 \mathrm{~s}$ have $240 \mathrm{MHz}$, 

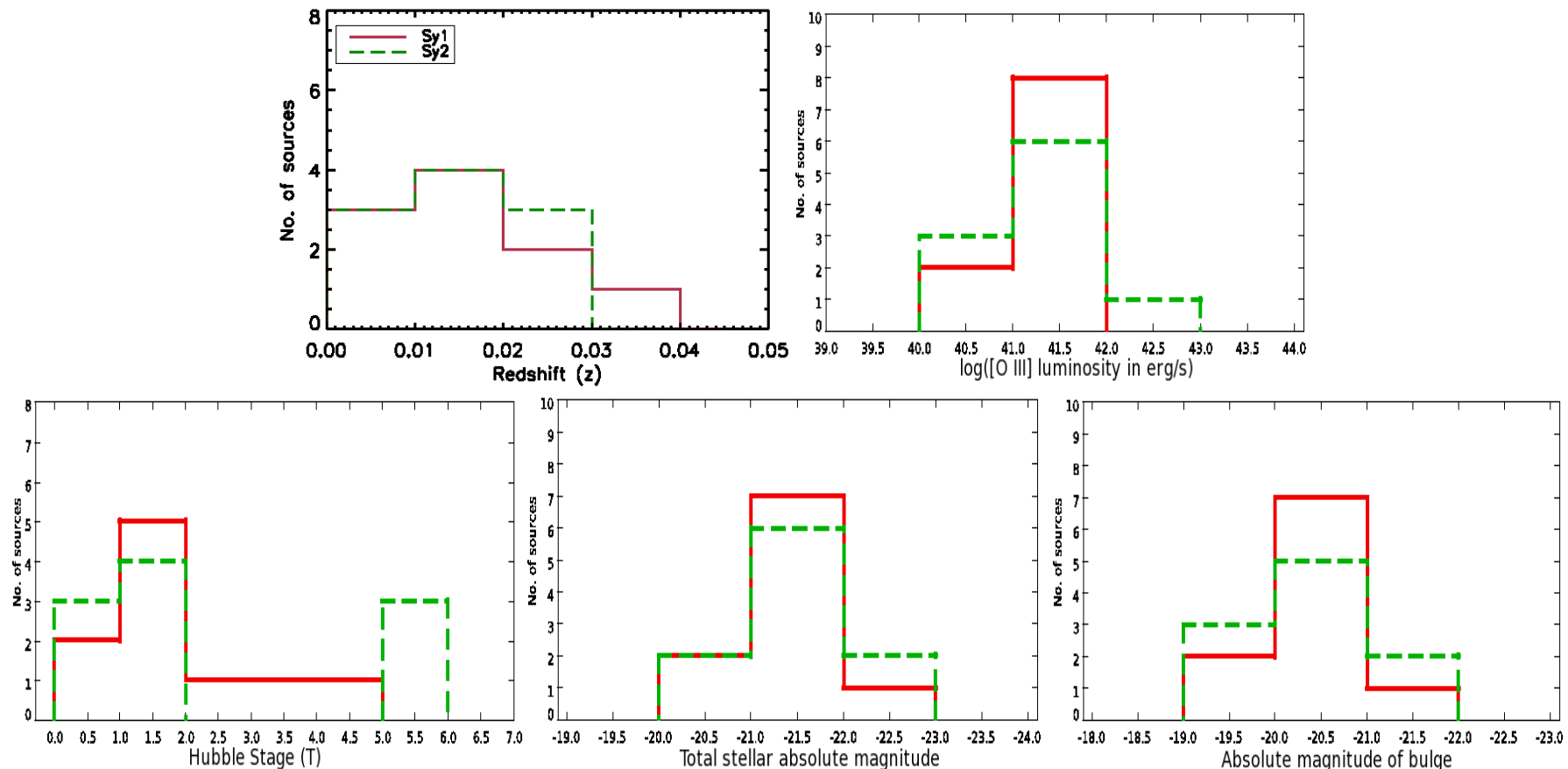

Fig. 1. Histograms showing matched distributions of Seyfert types $1 \mathrm{~s}$ and $2 \mathrm{~s}$ in redshift, [O III] $\lambda 5007 \AA$ A luminosity, Hubble type of the host galaxy, total stellar absolute magnitude of the host galaxy, and absolute magnitude of the bulge. The histograms for types $1 \mathrm{~s}$ and $2 \mathrm{~s}$ are plotted with red colored solid lines and green colored dashed lines, respectively.

$610 \mathrm{MHz}, 1.4 \mathrm{GHz}$, and $5.0 \mathrm{GHz}$ luminosities in the range of $L_{240 \mathrm{MHz}} \sim 7.33 \times 10^{28}-9.37 \times 10^{30} \mathrm{erg} \mathrm{s}^{-1} \mathrm{~Hz}^{-1}, L_{610 \mathrm{MHz}} \sim$ $8.26 \times 10^{28}-7.17 \times 10^{30} \mathrm{erg} \mathrm{s}^{-1} \mathrm{~Hz}^{-1}, L_{1.4 \mathrm{GHz}} \sim 5.07 \times$ $10^{28}-4.35 \times 10^{30} \mathrm{erg} \mathrm{s}^{-1} \mathrm{~Hz}^{-1}$, and $L_{5.0 \mathrm{GHz}} \sim 3.09 \times$ $10^{28}-1.48 \times 10^{30} \mathrm{erg} \mathrm{s}^{-1} \mathrm{~Hz}^{-1}$, respectively, with the median values $L_{240 \mathrm{MHz}}$, median $\sim 1.51 \times 10^{30} \mathrm{erg} \mathrm{s}^{-1} \mathrm{~Hz}^{-1}$, $L_{610 \mathrm{MHz}, \text { median }} \sim 6.75 \times 10^{29} \mathrm{erg} \mathrm{s}^{-1} \mathrm{~Hz}^{-1}, L_{1.4 \mathrm{GHz} \text {, median }} \sim$

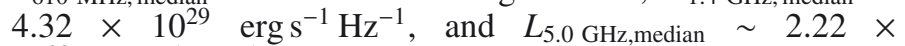
$10^{29} \mathrm{erg} \mathrm{s}^{-1} \mathrm{~Hz}^{-1}$, respectively. We note that the radio luminosity distributions for the two Seyfert subtypes at $240 \mathrm{MHz}$, $610 \mathrm{MHz}, 1.4 \mathrm{GHz}$, and $5.0 \mathrm{GHz}$ span a similar range with similar median values at the respective frequencies. The twosample Kolmogorov-Smirnov statistical test shows that there is $99 \%$ probability that the $L_{240 \mathrm{MHz}}$ and $L_{610 \mathrm{MHz}}$ distributions of the two Seyfert subtypes are drawn from the same parent population. The $1.4 \mathrm{GHz}$ and $5.0 \mathrm{GHz}$ luminosity distributions of the two Seyfert subtypes are also not statistically different (cf. Table 6). Our results for the comparison of radio luminosity complement the previous studies, which reported that the pc-scale nuclear radio luminosities at higher frequencies are similar for the two Seyfert subtypes (e.g., Lal et al. 2011).

\subsection{Radio spectra of Seyfert type 1s and type 2s}

Most of the previous studies on the spectral properties of Seyfert galaxies have been limited mainly to high-frequency regime $(\geq 1.4 \mathrm{GHz}$ ) (Barvainis et al. 1996; Rush et al. 1996; Morganti et al. 1999). Therefore, we aim to explore the nature of the radio spectra of Seyfert galaxies at relatively lower frequencies extending down to $240 \mathrm{MHz}$. And, we compare the radio spectra of Seyfert types $1 \mathrm{~s}$ and $2 \mathrm{~s}$ in the framework of the unification scheme. We discussed in the previous section that the radio luminosity/flux in Seyfert galaxies is independent of the orientation of the obscuring torus. This result implies that radio spectra should also be similar for the Seyfert types 1s and
Table 2. GMRT observational log.

\begin{tabular}{lccc}
\hline \hline $\begin{array}{l}\text { Source } \\
\text { name }\end{array}$ & $\begin{array}{c}\text { No. } \\
\text { of scans }\end{array}$ & $\begin{array}{c}\text { Phase } \\
\text { Cal }\end{array}$ & $\begin{array}{c}\text { Obs. } \\
\text { date }\end{array}$ \\
\hline MRK 6 & 3 & $\begin{array}{c}\text { Seyfert 1s } \\
0614+607\end{array}$ & 2008 August 09, 10 \\
NGC 3227 & 3 & $1111+199$ & 2008 August 09 \\
NGC 3516 & 4 & $1313+675$ & 2008 August 09, 10 \\
NGC 4151 & 2 & $1331+305$ & 2008 August 09 \\
MRK 766 & 3 & $1331+305$ & 2008 August 09, 10 \\
MRK 279 & 4 & $1313+675$ & 2008 August 09, 10 \\
NGC 5548 & 5 & $1331+305$ & 2008 August 09, 10 \\
ARK 564 & 3 & $2236+284$ & 2008 August 10 \\
NGC 7469 & 1 & $2212+018$ & 2008 August 10 \\
MRK 530 & 4 & $2212+018$ & 2008 August 10 \\
& & Seyfert 2s & \\
MRK 348 & 3 & $0137+331$ & 2008 August 10 \\
MRK 1 & 3 & $0137+331$ & 2008 August 10 \\
MRK 1066 & 3 & $0137+331$ & 2008 August 10 \\
NGC 2110 & 2 & $0607-085$ & 2008 August 10 \\
NGC 2273 & 3 & $0614+607$ & 2008 August 09, 10 \\
NGC 5252 & 4 & $1351-148$ & 2008 August 09, 10 \\
NGC 5728 & 2 & $1351-148$ & 2008 August 09 \\
NGC 7212 & 2 & $2212+018$ & 2008 August 10 \\
NGC 7682 & 2 & $2212+018$ & 2008 August 10 \\
MRK 533 & 2 & $2212+018$ & 2008 August 10 \\
\hline
\end{tabular}

$2 \mathrm{~s}$. This prediction of the unification scheme on the radio spectra have been examined in some of the previous studies reporting varying results. For instance, Edelson (1987) measured three point $(1.4 \mathrm{GHz}, 5.0 \mathrm{GHz}$, and $20 \mathrm{GHz})$ radio spectra of Seyfert galaxies and reported that types $1 \mathrm{~s}$ and $2 \mathrm{~s}$ show steep $\left(\alpha \sim-0.7, S_{v} \propto v^{\alpha}\right)$ radio spectra with type 1 s occasionally tend to show flatter or inverted spectra. However, Rush et al. (1996) reported that there is no significant difference between the average $1.4 \mathrm{GHz}-5.0 \mathrm{GHz}$ spectral indices of Seyfert types 1s 
Table 3. $610 \mathrm{MHz}$ radio image parameters.

\begin{tabular}{|c|c|c|c|c|c|c|c|c|c|}
\hline \multirow{3}{*}{$\begin{array}{l}\text { Sources } \\
\text { name }\end{array}$} & \multicolumn{4}{|c|}{ Map parameter } & \multicolumn{5}{|c|}{ Source parameter } \\
\hline & \multirow{2}{*}{$\begin{array}{l}\text { Scale } \\
\left(\mathrm{kpc} /{ }^{\prime \prime}\right)\end{array}$} & \multirow{2}{*}{$\begin{array}{c}\text { Beam size } \\
\left(\operatorname{arcsec}^{2}\right)\end{array}$} & \multirow{2}{*}{$\begin{array}{c}\text { PA } \\
\text { (deg) }\end{array}$} & \multirow{2}{*}{$\begin{array}{c}\text { rms noise } \\
(\mathrm{mJy} / \mathrm{b})\end{array}$} & \multirow{2}{*}{$\begin{array}{c}S_{\text {Peak }} \\
(\mathrm{mJy} / \mathrm{b})\end{array}$} & \multirow{2}{*}{$\begin{array}{c}S_{\text {Int. }} \\
(\mathrm{mJy})\end{array}$} & \multicolumn{3}{|c|}{ Fitted size } \\
\hline & & & & & & & $\begin{array}{c}\text { Maj. } \\
(\operatorname{arcsec})\end{array}$ & $\begin{array}{c}\text { Min. } \\
(\operatorname{arcsec})\end{array}$ & $\begin{array}{c}\text { PA } \\
(\text { deg })\end{array}$ \\
\hline & & & & Seyfert $1 \mathrm{~s}$ & & & & & \\
\hline MRK 6 & 0.377 & $10.20 \times 4.95$ & -28.38 & 1.65 & 476.2 & 502 & 10.42 & 5.06 & 150.8 \\
\hline NGC 3227 & 0.079 & $8.03 \times 6.44$ & -65.60 & 2.10 & 130.1 & 148 & 8.85 & 6.70 & 119.4 \\
\hline NGC 3516 & 0.179 & $7.51 \times 4.91$ & -12.96 & 1.20 & 13.5 & 25 & 10.44 & 7.38 & 4.0 \\
\hline \multirow{2}{*}{ NGC 4151} & 0.068 & $7.79 \times 5.04$ & -25.02 & 3.50 & 312.9 & 341.5 & 8.12 & 5.28 & 147.7 \\
\hline & & & & & 218.1 & 264.5 & 8.30 & 5.74 & 162.1 \\
\hline MRK 766 & 0.261 & $7.52 \times 4.63$ & -25.68 & 1.15 & 53.3 & 58.0 & 7.92 & 4.81 & 150.0 \\
\hline MRK 279 & 0.601 & $7.47 \times 5.27$ & 19.68 & 0.90 & 39.6 & 44 & 7.86 & 5.54 & 19.0 \\
\hline NGC 5548 & 0.344 & $6.31 \times 5.19$ & -10.90 & 0.95 & 19.7 & 56 & 14.33 & 7.11 & 158.5 \\
\hline ARK 564 & 0.491 & $7.68 \times 5.16$ & -45.09 & 1.00 & 61.0 & 62.5 & 7.62 & 4.91 & 132.3 \\
\hline NGC 7469 & 0.328 & $9.67 \times 5.36$ & -24.77 & 1.15 & 218.5 & 292 & 10.43 & 6.70 & 154.7 \\
\hline \multirow[t]{2}{*}{ MRK 530} & 0.584 & $7.18 \times 6.85$ & 35.11 & 0.60 & 37.1 & 40 & 7.28 & 7.18 & 97.0 \\
\hline & & & & Seyfert $2 s$ & & & & & \\
\hline MRK 348 & 0.302 & $6.28 \times 5.69$ & -14.78 & 2.00 & 480.9 & 499 & 6.34 & 5.81 & 157.0 \\
\hline MRK 1 & 0.320 & $6.27 \times 5.19$ & 0.87 & 0.75 & 109.8 & 115 & 6.31 & 5.32 & 177.3 \\
\hline MRK 1066 & 0.243 & $6.56 \times 5.21$ & 19.39 & 1.20 & 166.8 & 190 & 6.67 & 5.63 & 18.9 \\
\hline NGC 2110 & 0.158 & $6.88 \times 5.09$ & 5.93 & 1.20 & 494.4 & 554 & 7.68 & 5.11 & 9.0 \\
\hline NGC 2273 & 0.125 & $7.93 \times 4.77$ & -29.88 & 0.80 & 85.9 & 102 & 8.32 & 5.34 & 149.6 \\
\hline NGC 5252 & 0.458 & $10.68 \times 5.12$ & -22.24 & 0.70 & 18.9 & 21 & 10.56 & 5.66 & 159.6 \\
\hline \multirow[t]{2}{*}{ NGC 5728} & 0.189 & $11.59 \times 5.98$ & -22.67 & 0.75 & 25.6 & 34.3 & 12.60 & 7.46 & 154.7 \\
\hline & & & & & 17.5 & 39.4 & 16.10 & 9.39 & 159.9 \\
\hline NGC 7212 & 0.528 & $8.32 \times 4.84$ & -21.85 & 0.45 & 189.4 & 192 & 8.40 & 4.85 & 158.5 \\
\hline NGC 7682 & 0.344 & $10.20 \times 6.41$ & -23.64 & 0.55 & 92.3 & 98 & 10.21 & 6.82 & 150.8 \\
\hline MRK 533 & 0.572 & $6.80 \times 6.15$ & 46.30 & 1.20 & 340.7 & 364 & 6.98 & 6.26 & 44.1 \\
\hline
\end{tabular}

Table 4. $240 \mathrm{MHz}$ radio image parameters.

\begin{tabular}{|c|c|c|c|c|c|c|c|c|c|}
\hline \multirow{3}{*}{$\begin{array}{l}\text { Source } \\
\text { name }\end{array}$} & \multicolumn{4}{|c|}{ Map parameter } & \multicolumn{5}{|c|}{ Source parameter } \\
\hline & \multirow{2}{*}{$\begin{array}{l}\text { Scale } \\
\left(\mathrm{kpc} /{ }^{\prime \prime}\right)\end{array}$} & \multirow{2}{*}{$\begin{array}{l}\text { Beam size } \\
\left(\operatorname{arcsec}^{2}\right)\end{array}$} & \multirow{2}{*}{$\begin{array}{c}\text { PA } \\
(\operatorname{deg})\end{array}$} & \multirow{2}{*}{$\begin{array}{l}\text { rms noise } \\
(\mathrm{mJy} / \mathrm{b})\end{array}$} & \multirow{2}{*}{$\begin{array}{c}S_{\text {Peak }} \\
(\mathrm{mJy} / \mathrm{b})\end{array}$} & \multirow{2}{*}{$\begin{array}{c}S_{\text {Int. }} \\
(\mathrm{mJy})\end{array}$} & \multicolumn{3}{|c|}{ Fitted size } \\
\hline & & & & & & & $\begin{array}{c}\text { Maj. } \\
(\operatorname{arcsec})\end{array}$ & $\begin{array}{c}\text { Min. } \\
(\operatorname{arcsec})\end{array}$ & $\begin{array}{c}\text { PA } \\
\text { (deg) }\end{array}$ \\
\hline & & & & Seyfert $1 \mathrm{~s}$ & & & & & \\
\hline MRK 6 & 0.377 & $23.04 \times 11.36$ & -22.04 & 3.0 & 853.2 & 944.6 & 24.14 & 11.76 & 158.8 \\
\hline NGC 3227 & 079 & $7.46 \times 14.27$ & -50.31 & 5.6 & 117.0 & 170.0 & 32.61 & 18.02 & 125.8 \\
\hline NGC 3516 & 179 & $16.26 \times 11.44$ & -15.03 & 3.8 & 36.9 & 81 & 37.09 & 14.31 & 25.82 \\
\hline NGC 4151 & 0.068 & $17.27 \times 11.68$ & -14.00 & 12.0 & 1324.4 & 1348 & 17.10 & 11.64 & 165.9 \\
\hline MRK 766 & 0.261 & $18.13 \times 12.75$ & -18.95 & 7.5 & 74.8 & 85 & 16.20 & 9.90 & 152.5 \\
\hline MRK 279 & 0.601 & $16.09 \times 10.75$ & 28.19 & 3.5 & 80.3 & 96 & 17.69 & 11.52 & 31.7 \\
\hline NGC 5548 & 0.344 & $21.65 \times 15.17$ & -30.72 & 6.5 & 77.6 & 83 & 23.54 & 14.83 & 154.0 \\
\hline ARK 564 & 0.491 & $25.99 \times 13.81$ & -45.7 & 4.4 & 164.4 & 166 & 24.29 & 12.92 & 131.97 \\
\hline NGC 7469 & 0.328 & $38.01 \times 22.06$ & -1.01 & 9.5 & 793.5 & 807 & 37.86 & 20.68 & 177.1 \\
\hline MRK 530 & 0.584 & $38.13 \times 18.73$ & -17.42 & 9.0 & 107.3 & 130 & 46.92 & 21.46 & 156.4 \\
\hline & & & & Seyfert $2 s$ & & & & & \\
\hline MRK 348 & 0.302 & $18.52 \times 12.88$ & -35.86 & 5.6 & & 810 & 18.96 & 12.91 & 148.2 \\
\hline MRK 1 & 320 & $18.14 \times 12.64$ & -22.12 & 5.5 & 181.9 & 186 & 17.57 & 12.36 & 163.6 \\
\hline MRK 1066 & 0.243 & $16.89 \times 13.25$ & -1.87 & 6.0 & 288.6 & 300 & 17.25 & 13.53 & 177.0 \\
\hline NGC 2110 & 0.158 & $43.63 \times 34.50$ & -0.18 & 25.0 & 1498.5 & 1499 & 38.85 & 32.47 & 1.9 \\
\hline NGC 2273 & 0.125 & $19.04 \times 11.06$ & -26.39 & 4.0 & 113.8 & 127 & 20.84 & 11.53 & 152.3 \\
\hline NGC 5252 & 0.458 & $38.21 \times 12.37$ & -25.16 & 3.0 & 19.4 & 26.4 & 46.20 & 19.31 & 152.5 \\
\hline NGC 5728 & 0.189 & $27.42 \times 12.94$ & -19.36 & 3.0 & 22.8 & 40 & 37.62 & 19.20 & 131.6 \\
\hline NGC 7212 & 0.528 & $40.16 \times 29.61$ & -1.95 & 7.5 & 523.1 & 551 & 32.85 & 25.79 & 175.4 \\
\hline NGC 7682 & 0.344 & $46.13 \times 34.28$ & 0.87 & 7.5 & 227.9 & 228 & 38.68 & 29.23 & 17.14 \\
\hline MRK 533 & 0.572 & $17.87 \times 16.98$ & -33.83 & 9.0 & 639.2 & 842 & 20.01 & 19.04 & 66.3 \\
\hline
\end{tabular}

and 2s. Barvainis et al. (1996) studied four-point $(1.5 \mathrm{GHz}$, $4.9 \mathrm{GHz}, 8.4 \mathrm{GHz}$, and $14.9 \mathrm{GHz}$ ) radio spectra and reported that the radio spectral shapes of Seyfert galaxies and radio-quiet quasars are quite heterogeneous.

We obtained two-point spectral indices and integrated radio spectra over $240 \mathrm{MHz}$ to $5.0 \mathrm{GHz}$, of our sample Seyfert galaxies using $240 \mathrm{MHz}, 610 \mathrm{MHz}$ flux densities from our GMRT observations, 1.4 GHz flux densities from NVSS (Condon et al. 1998), and 5.0 GHz flux densities from the literature (e.g., Gallimore et al. 2006; Edelson 1987; Griffith et al. 1995). Since GMRT observations at $240 \mathrm{MHz}, 610 \mathrm{MHz}$, and NVSS observations at $1.4 \mathrm{GHz}$ are of relatively low resolution, we considered 
Table 5. Radio luminosities and spectral indices.

\begin{tabular}{|c|c|c|c|c|c|c|c|c|c|c|}
\hline $\begin{array}{l}\text { Source } \\
\text { name }\end{array}$ & $\begin{array}{c}L_{240 \mathrm{MHz}} \\
\left(\mathrm{erg} \mathrm{s}^{-1} \mathrm{~Hz}^{-1}\right)\end{array}$ & $\begin{array}{c}L_{610 \mathrm{MHz}} \\
\left(\mathrm{erg} \mathrm{s}^{-1} \mathrm{~Hz}^{-1}\right)\end{array}$ & $\begin{array}{c}S_{1.4 \mathrm{GHz}} \\
(\mathrm{mJy})\end{array}$ & $\begin{array}{c}L_{1.4 \mathrm{GHz}} \\
\left(\mathrm{erg} \mathrm{s}^{-1} \mathrm{~Hz}^{-1}\right)\end{array}$ & $\begin{array}{c}S_{5.0 \mathrm{GHz}} \\
(\mathrm{mJy})\end{array}$ & $\begin{array}{c}L_{5.0 \mathrm{GHz}} \\
\left(\mathrm{erg} \mathrm{s}^{-1} \mathrm{~Hz}^{-1}\right)\end{array}$ & $\alpha_{240 \mathrm{MHz}}^{610 \mathrm{MHz}}$ & $\alpha_{610 \mathrm{MHz}}^{1.4 \mathrm{GHz}}$ & $\alpha_{1.4 \mathrm{GHz}}^{5.0 \mathrm{GHz}}$ & $\alpha_{\text {int. }}$ \\
\hline \multicolumn{11}{|c|}{ Seyfert $1 \mathrm{~s}$} \\
\hline MRK 6 & $7.87 \times 10^{30}$ & $4.18 \times 10^{30}$ & 269.5 & $2.24 \times 10^{30}$ & $100.5^{\mathrm{K}}$ & $8.37 \times 10^{29}$ & -0.68 & -0.75 & -0.77 & -0.74 \\
\hline NGC 3227 & $6.09 \times 10^{28}$ & $5.30 \times 10^{28}$ & 97.5 & $3.49 \times 10^{28}$ & $35.0^{\mathrm{Ga}}$ & $1.25 \times 10^{28}$ & -0.15 & -0.50 & -0.80 & -0.53 \\
\hline NGC 3516 & $1.48 \times 10^{29}$ & $4.58 \times 10^{28}$ & 31.3 & $5.74 \times 10^{28}$ & $7.4^{\mathrm{Ga}}$ & $1.36 \times 10^{28}$ & -1.26 & +0.27 & -1.13 & -0.71 \\
\hline NGC 4151 & $2.71 \times 10^{29}$ & $1.22 \times 10^{29}$ & 359.6 & $7.24 \times 10^{28}$ & $128.0^{\mathrm{Ga}}$ & $2.58 \times 10^{28}$ & -0.86 & -0.63 & -0.81 & -0.76 \\
\hline MRK 766 & $3.28 \times 10^{29}$ & $2.24 \times 10^{29}$ & 38.1 & $1.47 \times 10^{29}$ & $20.4^{\mathrm{Ga}}$ & $7.86 \times 10^{28}$ & -0.41 & -0.51 & -0.49 & -0.47 \\
\hline MRK 279 & $2.03 \times 10^{30}$ & $9.28 \times 10^{29}$ & 23.2 & $4.89 \times 10^{29}$ & $7.4^{\mathrm{E}}$ & $1.56 \times 10^{29}$ & -0.84 & -0.77 & -0.90 & -0.84 \\
\hline NGC 5548 & $5.51 \times 10^{29}$ & $3.72 \times 10^{29}$ & 28.2 & $1.87 \times 10^{29}$ & $11.2^{\mathrm{Ga}}$ & $7.44 \times 10^{28}$ & -0.42 & -0.83 & -0.73 & -0.68 \\
\hline ARK 564 & $2.41 \times 10^{30}$ & $9.09 \times 10^{29}$ & 28.6 & $4.16 \times 10^{29}$ & $11.4^{\mathrm{L}}$ & $1.66 \times 10^{29}$ & -1.05 & -0.94 & -0.72 & -0.88 \\
\hline NGC 7469 & $4.74 \times 10^{30}$ & $1.71 \times 10^{30}$ & 180.5 & $1.06 \times 10^{30}$ & $61.6^{\mathrm{Ga}}$ & $3.62 \times 10^{29}$ & -1.09 & -0.58 & -0.84 & -0.82 \\
\hline MRK 530 & $2.56 \times 10^{30}$ & $7.88 \times 10^{29}$ & 24.4 & $4.80 \times 10^{29}$ & $11.5^{\mathrm{E}}$ & $2.26 \times 10^{29}$ & -1.26 & -0.59 & -0.59 & -0.77 \\
\hline \multicolumn{11}{|c|}{ Seyfert $2 s$} \\
\hline MRK 348 & $4.17 \times 10^{30}$ & $2.57 \times 10^{30}$ & 292.2 & $1.51 \times 10^{30}$ & $801.7^{\mathrm{Ga}}$ & $4.13 \times 10^{29}$ & -0.52 & -0.64 & +0.79 & -0.58 \\
\hline MRK 1 & $1.09 \times 10^{30}$ & $6.75 \times 10^{29}$ & 75.4 & $4.43 \times 10^{29}$ & $32.0^{\mathrm{GC}}$ & $1.88 \times 10^{29}$ & -0.52 & -0.51 & -0.67 & -0.58 \\
\hline MRK 1066 & $9.83 \times 10^{29}$ & $6.23 \times 10^{29}$ & 100.4 & $3.29 \times 10^{29}$ & $35.0^{\mathrm{GC}}$ & $1.15 \times 10^{29}$ & -0.49 & -0.77 & -0.83 & -0.72 \\
\hline NGC 2110 & $2.17 \times 10^{30}$ & $8.01 \times 10^{29}$ & 298.8 & $4.32 \times 10^{29}$ & $165.0^{\mathrm{Gr}}$ & $2.38 \times 10^{29}$ & -1.07 & -0.74 & -0.47 & -0.72 \\
\hline NGC 2273 & $1.03 \times 10^{29}$ & $8.26 \times 10^{28}$ & 62.6 & $5.07 \times 10^{28}$ & $44.0^{\mathrm{GC}}$ & $3.56 \times 10^{28}$ & -0.24 & -0.59 & -0.28 & -0.37 \\
\hline NGC 5252 & $3.31 \times 10^{29}$ & $2.58 \times 10^{29}$ & 16.3 & $2.00 \times 10^{29}$ & $18.1^{\mathrm{E}}$ & $2.22 \times 10^{29}$ & -0.27 & -0.30 & +0.08 & -0.14 \\
\hline NGC 5728 & $7.33 \times 10^{28}$ & $1.36 \times 10^{29}$ & 70.0 & $1.28 \times 10^{29}$ & $17.5^{\mathrm{S}}$ & $3.09 \times 10^{28}$ & +0.66 & -0.07 & -1.09 & $\ldots$ \\
\hline NGC 7212 & $9.37 \times 10^{30}$ & $3.27 \times 10^{30}$ & $128.0^{\mathrm{W}}$ & $2.18 \times 10^{30}$ & $46.0^{\mathrm{Gr}}$ & $7.83 \times 10^{29}$ & -1.13 & -0.49 & -0.80 & -0.79 \\
\hline NGC 7682 & $1.51 \times 10^{30}$ & $6.51 \times 10^{29}$ & 59.8 & $3.97 \times 10^{29}$ & $24.6^{\mathrm{E}}$ & $1.63 \times 10^{29}$ & -0.91 & -0.59 & -0.70 & -0.72 \\
\hline MRK 533 & $1.66 \times 10^{31}$ & $7.17 \times 10^{30}$ & 220.9 & $4.35 \times 10^{30}$ & $75.1^{\mathrm{E}}$ & $1.48 \times 10^{30}$ & -0.90 & -0.60 & -0.85 & -0.78 \\
\hline
\end{tabular}

Notes. 1.4 GHz flux densities are from NVSS catalog (Condon et al. 1998) except for NGC 7212 for which NVSS data are unavailable.

References. (K) Kharb et al. (2006); (E) Edelson (1987); (Ga) Gallimore et al. (2006); (GC) Gregory \& Condon (1991); (Gr) Griffith et al. (1995); (L) Lal et al. (2011); (S) Schommer et al. (1988); (W) White \& Becker (1992).
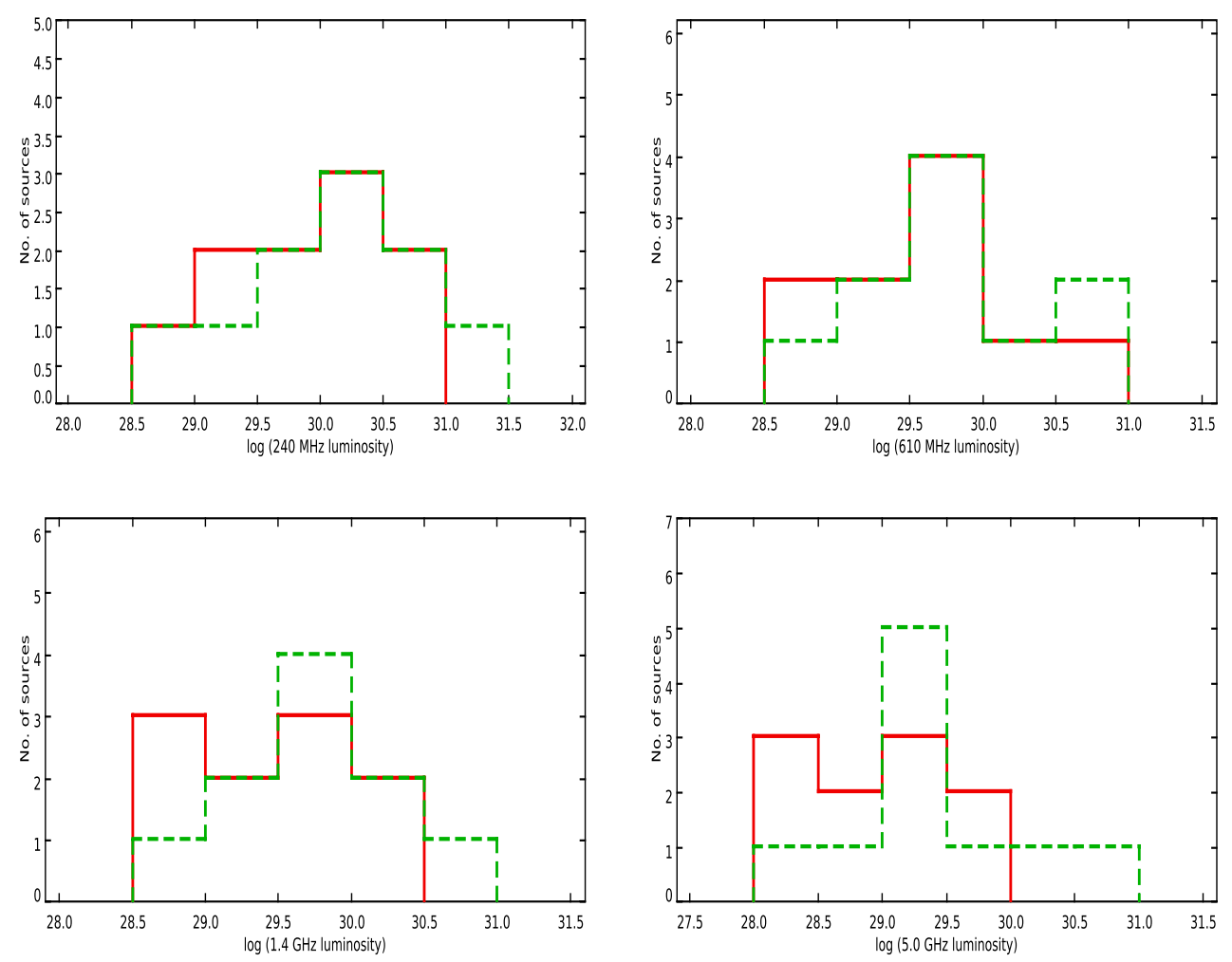

Fig. 3. Histograms of radio luminosities at $240 \mathrm{MHz}, 610 \mathrm{MHz}$, $1.4 \mathrm{GHz}$, and $5.0 \mathrm{GHz}$ for Seyfert types $1 \mathrm{~s}$ and $2 \mathrm{~s}$. The histograms for types $1 \mathrm{~s}$ and $2 \mathrm{~s}$ are plotted with red solid lines and green dashed lines, respectively.

$5 \mathrm{GHz}$ flux density measured with low-resolution observations, i.e., VLA in "D" configuration (Gallimore et al. 2006; Edelson 1987) or from single-dish Green Bank and Parkes radio telescopes (Griffith et al. 1995; Gregory \& Condon 1991).

Table 5 lists the two-point radio spectral indices measured between $240 \mathrm{MHz}$ to $610 \mathrm{MHz}\left(\alpha_{240}^{610 \mathrm{MHz}}\right), 610 \mathrm{MHz}$ to $1.4 \mathrm{MHz}$ $\left(\alpha_{610 \mathrm{MHz}}^{1.4 \mathrm{GHz}}\right)$, and $1.4 \mathrm{GHz}$ to $5.0 \mathrm{GHz}\left(\alpha_{1.4 \mathrm{GHz}}^{5.0 \mathrm{GHz}}\right)$, as well as the integrated radio spectral indices $\left(\alpha_{\text {int }}\right)$ estimated using four flux density points. Figure 4 shows the four-point $(240 \mathrm{MHz}$, $610 \mathrm{MHz}, 1.4 \mathrm{GHz}$, and $5.0 \mathrm{GHz}$ ) radio spectra of all our sample Seyfert galaxies. The integrated radio spectral index $\left(\alpha_{\text {int }}\right)$ is obtained by fitting all four spectral points with a linear chi-square 
fit. The slope of the line fit on a logarithmic scale gives the index of the powerlaw spectrum $\left(\mathrm{S}_{v} \propto v^{\alpha}\right)$. Figure 5 shows the distributions of two-points $\alpha_{240 \mathrm{MHz}}^{610 \mathrm{MH}}, \alpha_{610 \mathrm{MHz}}^{1.4 \mathrm{GHz}}, \alpha_{1.4 \mathrm{GHz}}^{5.0 \mathrm{GH}}$, and integrated $\alpha_{\text {int }}$ spectral indices for Seyfert types 1 s and $2 \mathrm{~s}$ of our sample. We note that spectral index distributions for the two subtypes span over similar ranges with median spectral indices $\alpha_{240 \mathrm{MHz}}^{610 \mathrm{MHz}} \simeq-0.83, \alpha_{610 \mathrm{MHz}}^{1.4 \mathrm{GHz}} \simeq-0.59, \alpha_{1.4 \mathrm{GHz}}^{5.0 \mathrm{GHz}} \simeq-0.77, \alpha_{\mathrm{int}} \simeq$ -0.74 for type $1 \mathrm{~s}$, and $\alpha_{240 \mathrm{MHz}}^{610 \mathrm{MHz}} \simeq-0.52, \alpha_{610 \mathrm{MHz}}^{1.4 \mathrm{GHz}} \simeq-0.59$, $\alpha_{1.4 \mathrm{GHz}}^{5.0 \mathrm{GHz}} \simeq-0.67, \alpha_{\text {int }} \simeq-0.72$ for type $2 \mathrm{~s}$ (cf., Table 6). The statistical comparison using two-sample KS test shows that the distributions of spectral indices for the two Seyfert subtypes are not very different (cf., Table 6).

Since the synthesized beam size is different at different frequencies (e.g., $\sim 20^{\prime \prime}-40^{\prime \prime}$ at $240 \mathrm{MHz}, \sim 8^{\prime \prime}-10^{\prime \prime}$ at $610 \mathrm{MHz}$, $\sim 45^{\prime \prime}$ at $1.4 \mathrm{GHz}$, and $\sim 20^{\prime \prime}$ or larger at $5.0 \mathrm{GHz}$ ), it may result an error in the estimated spectral index values. For example, the larger NVSS synthesized beam $\left(\sim 45^{\prime \prime}\right)$ at $1.4 \mathrm{GHz}$ compared to the GMRT synthesized beam $\left(\sim 8^{\prime \prime}-10^{\prime \prime}\right)$ at $610 \mathrm{MHz}$ may result less steep spectrum than actual. We assume a conservative fiducial error values of $15 \%$ at $240 \mathrm{MHz}$ flux density, $10 \%$ at $610 \mathrm{MHz}$ flux density, $7 \%$ at $1.4 \mathrm{GHz}$ flux density, and $7 \%$ at 5.0 GHz flux density. The assumed errors in flux densities can result $\sim 8 \%, \sim 5 \%, \sim 4 \%$, and $\sim 7 \%$ errors in $\alpha_{240 \mathrm{MHz}}^{610 \mathrm{MHz}}, \alpha_{610 \mathrm{MHz}}^{1.4 \mathrm{GHz}}$, $\alpha_{1.4 \mathrm{GHz}}^{5.0 \mathrm{GHz}}$, and $\alpha_{\mathrm{int}}$, respectively. Also, non-simultaneous observations may contribute to error in $\alpha_{610 \mathrm{MHz}}^{1.4 \mathrm{GHz}}, \alpha_{1.4 \mathrm{GHz}}^{5.0 \mathrm{GHz}}$, and $\alpha_{\text {int }}$ estimates, if the source flux density varies in between two observations. Moreover, the estimates of $\alpha_{240 \mathrm{MHz}}^{610 \mathrm{MH}}$ are free of the error due to non-simultaneity, since $240 \mathrm{MHz}$ and $610 \mathrm{MHz}$ flux densities are from simultaneous GMRT observations.

In MRK 348, the 5.0 GHz flux density $(\sim 807.1 \mathrm{mJy}$ from VLA "D" array observations Gallimore et al. 2006) is much higher than expected from a powerlaw spectral shape determined by $240 \mathrm{MHz}, 610 \mathrm{MHz}$, and $1.4 \mathrm{GHz}$ flux densities. Therefore, we consider $5.0 \mathrm{GHz}$ flux density as an outlier in fitting the radio spectrum of MRK 348. The unusual high flux density at $5.0 \mathrm{GHz}$ can be attributed to strong variability, since the core of MRK 348 is variable at $5.0 \mathrm{GHz}$ on a scale of months (Neff \& de Bruyn 1983; Ulvestad et al. 1999). However, it is worth noting that except in a few cases (Neff \& de Bruyn 1983; Wrobel 2000; Falcke et al. 2000), most of the Seyfert galaxies show little radio variability over the period of a few years (Edelson 1987; Mundell et al. 2009), and therefore our statistical results are not expected to be affected much by variability.

We note that most of the Seyfert galaxies in our sample have steep integrated radio spectra $\left(\alpha_{\text {int }} \sim-0.65\right.$ to -0.85$)$, except NGC 5252 and NGC 5728, which show flat and inverted spectrum, respectively. Since NGC 5728 shows inverted spectrum over $240 \mathrm{MHz}$ to $5.0 \mathrm{GHz}$, we do not obtain integrated spectral index measured by linear chi-square fit. There are a few sources e.g., NGC 3227, MRK 1066, and NGC 5548, which show hints of spectral flattening at lower frequencies, while a few cases (e.g., NGC 3516, MRK 530) show hints of spectral steepening at lower frequencies. The steep radio spectrum can be interpreted as emission produced via synchrotron radiation and associated to relatively extended structures. The integrated radio spectral shape of a Seyfert galaxy can be attributed to the combined contributions of all the radio emitting components. In low-resolution radio observations, the radio emission from Seyfert galaxies can have contributions from four components, i.e., a partially opaque synchrotron emission from a compact parsec-scale nuclear core, optically thin synchrotron emission from an extended component powered by AGN, optically thin synchrotron emission from star-forming regions present in the host galaxy disk, and a circumnuclear starburst emission (Wilson et al. 1991). The relative fraction of these emitting components may vary from one source to another and, in turn, may change the spectral shape.

Previous radio studies have shown that the total radio emission in Seyfert galaxies may have contributions from host galaxy disk and from starburst regions, but the radio emission in most of the Seyfert galaxies is dominated by nuclear radio emission characterized by high brightness temperature, steep spectrum, and non-thermal emission (Kukula et al. 1998). The extended emission powered by AGN, as well as star formation, gives rise steep spectrum, and therefore it is difficult to conclude whether emission is powered by either AGN or starburst by using only spectral shape information. In case of sources showing flat or inverted spectra, the total radio emission is likely to be dominated by the compact nuclear core that is partially opaque to synchrotron emission (Kukula et al. 1998). The compact nuclear radio emission characterized by high brightness temperature $\left(\sim 10^{8} \mathrm{~K}\right)$ and the inverted spectrum seen in some of Seyfert galaxies is indicative of the synchrotron self-absorption close to the jet-emanating region (Mundell et al. 2000); however, the free-free absorption by thermal, ionized gas in the vicinity of the nucleus might also be sufficient to flatten the intrinsically steeper synchrotron spectra (Ho \& Ulvestad 2001).

\subsection{Radio morphologies of Seyfert types $1 \mathrm{~s}$ and 2s}

High-resolution radio observations of Seyfert galaxies show parsec-scale nuclear emission often accompanied by a jet-like elongated feature, which is believed to represent outflowing radio emitting plasma from AGN, in the form of jets, bubbles or plasmoids (Wilson \& Ulvestad 1982b; Thean et al. 2001; Lal et al. 2004). These are thought to be small-scale, low-power versions of the large-scale jets seen in radio-loud AGNs. Radio observations of relatively lower resolution reveal that several Seyfert galaxies exhibit extended radio emission up to a few kpc (Baum et al. 1993; Colbert et al. 1996; Gallimore et al. 2006). The comparison of the radio sizes of two Seyfert subtypes can be used to test the unification scheme, provided that the radio emission is primarily due to linear jet-like outflows emanating from AGN and these radio structures are well resolved. According to the orientation-based unification scheme, Seyfert type 1s are expected to show smaller projected radio source sizes than type $2 \mathrm{~s}$, since radio jets in type $1 \mathrm{~s}$ are expected to lie along the line-ofsight to the observer, hence to be foreshortened. Earlier studies that attempted to test this prediction reported that Seyfert type $2 \mathrm{~s}$ are larger in radio than type 1s (e.g., Ulvestad \& Wilson 1984a). After controlling for the strengths of the radio sources, Ulvestad \& Wilson (1989) found that the differences in radio sizes of two subtypes are not statistically significant. Using a sample of Seyfert galaxies based on $60 \mu \mathrm{m}$, Schmitt et al. (2001) report that the radio sizes of type $2 \mathrm{~s}$ are systematically larger than type $1 \mathrm{~s}$, while, Ulvestad \& Ho (2001) report that type 1s appears to be larger than type $2 \mathrm{~s}$, which is inconsistent with the unification scheme.

We carried out our GMRT observations with the primary aim of studying the radio spectra of Seyfert galaxies at lower frequencies. Moreover, we attempted to study the low-frequency radio morphologies of our sample Seyfert galaxies. Figure 2 shows the $240 \mathrm{MHz}$ and $610 \mathrm{MHz}$ radio contour images overlaid on their Digital Sky Survey (DSS) optical images for all of our sample Seyfert galaxies. Tables 3.0 and 4.0 list the map parameters (i.e., synthesized beam size, its position angle and noise rms) and source parameters (i.e., total flux density $\left(S_{\text {int }}\right)$, peak flux 

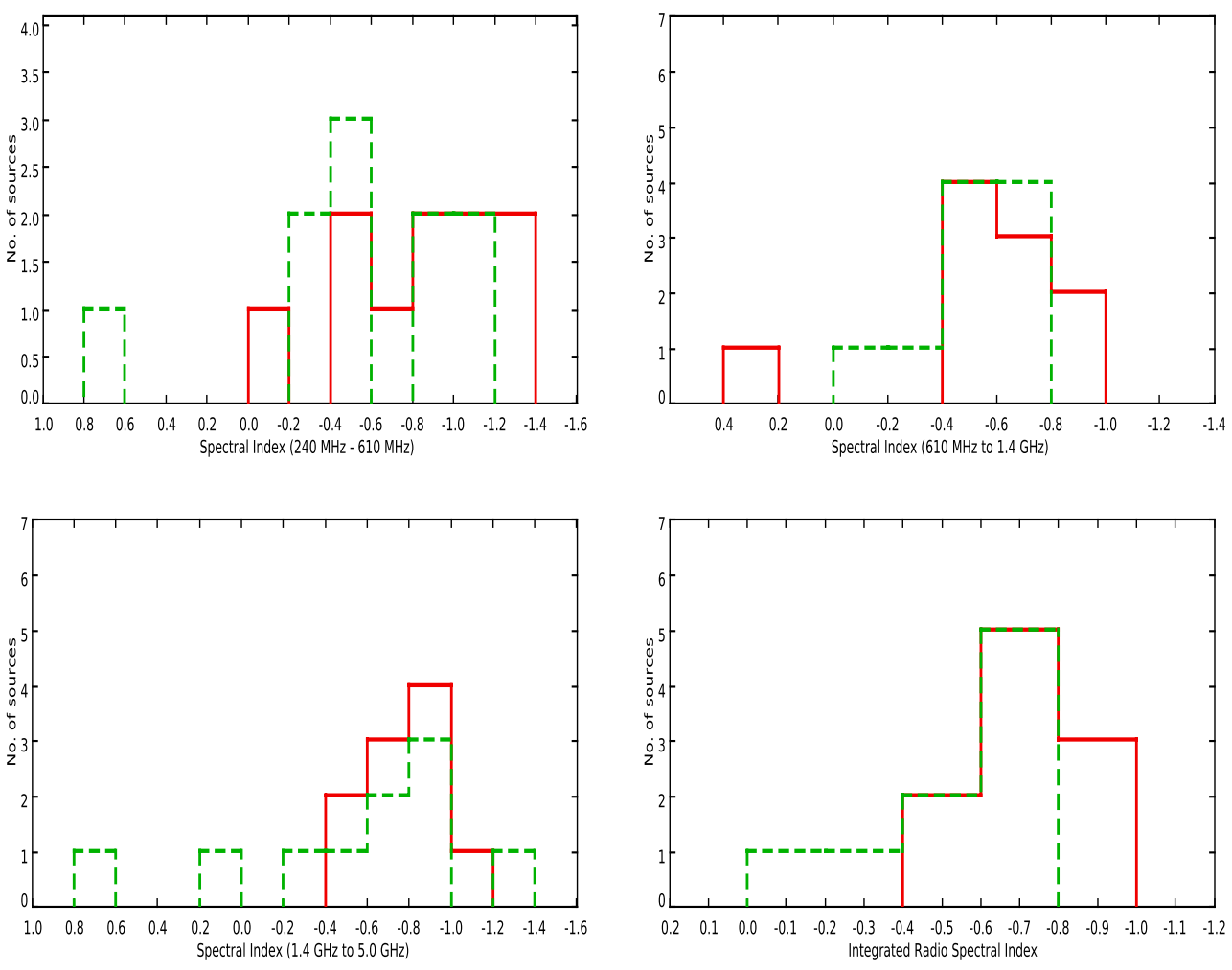

Fig. 5. Histograms of two-point spectral indices $\alpha_{240 \mathrm{MHz}}^{610 \mathrm{MHz}}, \alpha_{610 \mathrm{MHz}}^{1.4 \mathrm{GHz}}$, $\alpha_{50 \mathrm{GHz}}^{1.4 \mathrm{GHz}}$, and integrated spectral in$\operatorname{dex}\left(\alpha_{\text {int }}\right)$ for the two subtypes of our sample Seyfert galaxies. The histograms for types $1 \mathrm{~s}$ and $2 \mathrm{~s}$ are plotted with red solid lines and green dashed lines, respectively.

Table 6. Comparison of radio luminosities and spectral indices of Seyfert types 1s and 2s.

\begin{tabular}{|c|c|c|c|c|c|c|c|c|c|c|c|}
\hline \multirow[t]{3}{*}{ Distribution } & \multicolumn{8}{|c|}{ Statistical parameters } & \multicolumn{2}{|c|}{ KS test } & \multirow[t]{3}{*}{ F.D. Ref. } \\
\hline & \multicolumn{4}{|c|}{ Seyfert type $1 \mathrm{~s}$} & \multicolumn{4}{|c|}{ Seyfert type $2 \mathrm{~s}$} & & & \\
\hline & $\min$ & $\max$ & SD & median & $\min$ & $\max$ & SD & median & $D$ & $p$-value & \\
\hline $\log L_{240 \mathrm{MHz}}$ & 28.78 & 30.90 & 0.66 & 30.31 & 28.87 & 31.22 & 0.73 & 30.18 & 0.2 & 0.99 & 1 \\
\hline $\log L_{610 \mathrm{MHz}}$ & 28.66 & 30.62 & 0.61 & 29.90 & 28.92 & 30.86 & 0.58 & 29.83 & 0.2 & 0.99 & 1 \\
\hline $\log L_{14 \mathrm{GHz}}$ & 28.54 & 30.35 & 0.55 & 29.62 & 28.70 & 30.64 & 0.55 & 29.64 & 0.3 & 0.79 & 2 \\
\hline $\log L_{5.0 \mathrm{GHz}}$ & 28.10 & 29.92 & 0.57 & 29.19 & 28.51 & 30.62 & 0.63 & 29.35 & 0.3 & 0.79 & 3 \\
\hline$\alpha_{240 \mathrm{MHz}}^{610 \mathrm{MHz}}$ & -1.26 & -0.15 & 0.36 & -0.83 & -1.13 & +0.66 & 0.50 & -0.52 & 0.3 & 0.79 & 1 \\
\hline$\alpha_{610 \mathrm{MHz}}^{1.4 \mathrm{GHz}}$ & -0.94 & +0.27 & 0.31 & -0.59 & -0.77 & -0.07 & 0.20 & -0.59 & 0.3 & 0.79 & 1,2 \\
\hline$\alpha_{1.4 \mathrm{GHz}}^{5.0 \mathrm{GHz}}$ & -1.13 & -0.49 & 0.16 & -0.77 & -1.38 & +0.79 & 0.57 & -0.67 & 0.4 & 0.42 & 2,3 \\
\hline$\alpha_{\text {Int. }}$ & 0.88 & -0.47 & 0.12 & -0.74 & -0.79 & -0.14 & 0.20 & -0.72 & 0.38 & 0.51 & $1,2,3$ \\
\hline
\end{tabular}

Notes. Flux density (F.D.) references: (1) our GMRT observations; (2) NRAO VLA Sky Survey (NVSS); (3) Literature (Gallimore et al. 2006; Edelson 1987; Gregory \& Condon 1991; Griffith et al. 1995). The adopted conservative error values of $15 \%, 10 \%, 7 \%$, and $7 \%$ for $S_{240} \mathrm{MHz}$, $S_{610 \mathrm{MHz}}, S_{1.4 \mathrm{GHz}}$, and $S_{5.0 \mathrm{GHz}}$, respectively, render $\sim 8 \%, \sim 5 \%, \sim 4 \%$, and $\sim 7 \%$ errors in $\alpha_{240 \mathrm{MHz}}^{610 \mathrm{MHz}}, \alpha_{610 \mathrm{MHz}}^{1.4 \mathrm{GHz}}, \alpha_{1.4 \mathrm{GHz}}^{5.0}$, and $\alpha_{\mathrm{int}}$, respectively. Radio luminosities are in units of ergs s${ }^{-1} \mathrm{~Hz}^{-1}$. Kolmogorov-Smirnov (KS) two-sample test examines the hypothesis that two samples comes from same distribution. $D=\operatorname{Sup} \mathrm{x}|\mathrm{S} 1(\mathrm{x})-\mathrm{S} 2(\mathrm{x})|$ is the maximum difference between the cumulative distributions of two samples $\mathrm{S} 1(\mathrm{x})$ and $\mathrm{S} 2(\mathrm{x})$, respectively.

density $\left(S_{\text {peak }}\right)$, and source fitted sizes) for all 20 Seyfert galaxies at $610 \mathrm{MHz}$ and $240 \mathrm{MHz}$, respectively. In our $610 \mathrm{MHz}$ radio maps, the typical noise $\mathrm{rms}$ and resolution are $\sim 1.0 \mathrm{mJy} / \mathrm{beam}$ and $\sim 8^{\prime \prime}-10^{\prime \prime}$, respectively, while at $240 \mathrm{MHz}$, the typical noise $\mathrm{rms}$ is $\sim 7.5 \mathrm{mJy} /$ beam and resolution is $\sim 18^{\prime \prime}-40^{\prime \prime}$. We used the AIPS task "JMFIT" to measure the angular size of the source. Most of our sample sources are fitted with only one Gaussian component. The radio emission at $610 \mathrm{MHz}$ is fitted with a single Gaussian in all of our sample sources except for NGC 4151 and NGC 5728. In NGC 4151 and NGC 5728, the $610 \mathrm{MHz}$ radio emission has two distinct components that are fitted with two elliptical Gaussian components. At $610 \mathrm{MHz}$, several of our sample sources, i.e., NGC 3516, NGC 4151, NGC 5548, NGC 7469, MRK 1066, and NGC 5728 have fitted
Gaussian sizes larger than their synthesized beams which indicates the possibility of the existence of kpc-scale extended radio emission in these sources. Indeed, some of our sample sources e.g., NGC 4151, MRK 766, MRK 348, NGC 5548, and NGC 7469 are reported to possess kpc-scale extended emission at 5.0 GHz (Gallimore et al. 2006). At $240 \mathrm{MHz}$, all our sources are fitted with a single Gaussian component with sizes similar to their synthesized beams. Thus all our sample Seyferts can be interpreted as unresolved point sources at $240 \mathrm{MHz}$. We did not compare the radio sizes of the two Seyfert subtypes because most of our sample sources are seen as unresolved point sources at $240 \mathrm{MHz}$ and $610 \mathrm{MHz}$. Moreover, both Seyfert types 1s and $2 \mathrm{~s}$ in our sample show similar likelihood of being represented as unresolved point sources at the given sensitivity and resolution 
of our $240 \mathrm{MHz}$ and $610 \mathrm{MHz}$ GMRT observations. The lowfrequency radio emission in Seyfert galaxies may have contributions from star-forming regions or starburst other than the AGN. But our marginally resolved or unresolved radio images do not allow us to put any constraint on the relative contributions from different emitting components.

\section{Conclusions}

We present low-frequency radio images and spectra of our sample of 20 Seyfert galaxies where the sample is based on orientation-independent isotropic properties. In our sample the two subtypes have matched distributions in orientationindependent parameters that allow us to assume that the two subtypes are intrinsically similar within the framework of the unification scheme. Here we outline following conclusions from our study.

1. This work is the first attempt, to our knowledge, of a systematic study of low-frequency radio imaging and spectral properties of a well-defined sample of Seyfert galaxies.

2. The $240 \mathrm{MHz}, 610 \mathrm{MHz}, 1.4 \mathrm{GHz}$, and $5.0 \mathrm{GHz}$ radio luminosities of our sample of Seyfert galaxies are in the range of $\sim 10^{28}-10^{31} \mathrm{erg} \mathrm{s}^{-1}$. The $240 \mathrm{MHz}, 610 \mathrm{MHz}, 1.4 \mathrm{GHz}$, and $5.0 \mathrm{GHz}$ radio luminosity distributions of Seyfert type $1 \mathrm{~s}$ and type 2 s span over a similar range with similar median values at the respective frequencies. The two-sample KS test shows that $240 \mathrm{MHz}, 610 \mathrm{MHz}, 1.4 \mathrm{GHz}$, and $5.0 \mathrm{GHz}$ radio luminosity distributions of the two Seyfert subtypes are similar with a statistically significant probability.

3. We obtained integrated radio spectra over $240 \mathrm{MHz}$ to $5.0 \mathrm{GHz}$, of all our 20 Seyfert galaxies. We find that the distributions of two point spectral indices $\left(\alpha_{240 \mathrm{MHz}}^{610}, \alpha_{610 \mathrm{MHz}}^{1.4 \mathrm{GHz}}\right.$, $\alpha_{1.4 \mathrm{GHz}}^{5.0 \mathrm{GHz}}$ ), as well as integrated spectral index for the two subtypes, span over a similar range with median values $\alpha_{240 \mathrm{MHz}}^{610 \mathrm{MHz}} \simeq-0.83, \alpha_{610 \mathrm{MHz}}^{1.4 \mathrm{GHz}} \simeq-0.59, \alpha_{1.4 \mathrm{GHz}}^{5.0 \mathrm{GHz}} \simeq-0.77$, $\alpha_{\text {int }} \simeq-0.74$ for type $1 \mathrm{~s}$, and $\alpha_{240 \mathrm{MHz}}^{610 \mathrm{MHz}} \simeq-0.52, \alpha_{610 \mathrm{MHz}}^{1.4 \mathrm{GHz}} \simeq$ $-0.59, \alpha_{1.4 \mathrm{GHz}}^{5.0 \mathrm{GHz}}-0.67, \alpha_{\mathrm{int}} \simeq-0.72$ for type $2 \mathrm{~s}$. The two-sample KS test shows that the distributions of spectral indices for the two Seyfert subtypes are not statistically different.

4. We noted that most of the Seyfert galaxies in our sample have steep integrated radio spectra $\left(\alpha_{\text {int }} \sim-0.7\right)$, except for NGC 5252 and NGC 5728, which show flat and inverted spectra, respectively. The average steep radio spectral index is consistent with the previous studies (Morganti et al. 1999) and can be explained as optically thin synchrotron emission.

5. The $610 \mathrm{MHz}$ radio images of our sample sources can generally be represented as unresolved point sources wherein the radio emission is fitted with a single Gaussian component in all of our sample sources, except for NGC 4151 and NGC 5728, where, the $610 \mathrm{MHz}$ radio emission shows two distinct components.

6. At $240 \mathrm{MHz}$, all our sample sources show radio emission as an unresolved point source, since the radio emission is fitted with a single Gaussian component with sizes similar to their synthesized beams.

7. Radio images in our snapshot GMRT 240/610 MHz observations remain mostly unresolved. This does not allow us to compare the radio sizes of the two Seyfert subtypes in the framework of the unification scheme. Moreover, both Seyfert type $1 \mathrm{~s}$ and type $2 \mathrm{~s}$ in our sample show similar likelihood of being represented as unresolved point sources at the given sensitivity and resolution of our $240 \mathrm{MHz}$ and $610 \mathrm{MHz}$ GMRT observations.

8. In our study we have shown that the multifrequency radio properties i.e., luminosity and spectral distributions over $240 \mathrm{MHz}$ to $5 \mathrm{GHz}$ of our sample Seyfert galaxies are consistent with the orientation and obscuration-based unification scheme. In the Appendix, we discuss 240/610 MHz GMRT radio properties and its comparison to the radio observations reported in the literature for all the individual sources in our sample. Our results on the low-frequency radio properties are complementary and consistent with the reported highfrequency radio observations.

Acknowledgements. We thank the staff of GMRT who have made these observations possible. GMRT is run by the National Centre for Radio Astrophysics of the Tata Institute of Fundamental Research. V.S. would like to thank Dr. Chiranjib Konar for helpful discussions on $240 \mathrm{MHz}$ GMRT data reduction. Also, this research made use of the NASA/IPAC Extragalactic Database (NED), which is operated by the Jet Propulsion Laboratory, California Institute of Technology, under contract with the National Aeronautics and Space Administration.

\section{References}

Antón, S., Thean, A. H. C., Pedlar, A., \& Browne, I. W. A. 2002, MNRAS, 336, 319

Antonucci, R. 1993, ARA\&A, 31, 473

Antonucci, R. 2002, in AGN Surveys, eds. R. F. Green, E. Y. Khachikian, \& D. B. Sanders, IAU Colloq. 184, ASP Conf. Ser., 284, 147

Antonucci, R. R. J., \& Miller, J. S. 1985, ApJ, 297, 621

Barvainis, R., Lonsdale, C., \& Antonucci, R. 1996, AJ, 111, 1431

Baum, S. A., O’Dea, C. P., Dallacassa, D., de Bruyn, A. G., \& Pedlar, A. 1993, ApJ, 419, 553

Beckmann, V., Soldi, S., Ricci, C., et al. 2009, A\&A, 505, 417

Booler, R. V., Pedlar, A., \& Davies, R. D. 1982, MNRAS, 199, 229

Buchanan, C. L., Gallimore, J. F., O’Dea, C. P., et al. 2006, AJ, 132, 401

Cappi, M., Panessa, F., Bassani, L., et al. 2006, A\&A, 446, 459

Colbert, E. J. M., Baum, S. A., Gallimore, J. F., O’Dea, C. P., \& Christensen, J. A. 1996, ApJ, 467, 551

Condon, J. J., Cotton, W. D., Greisen, E. W., et al. 1998, AJ, 115, 1693

Dadina, M. 2008, A\&A, 485, 417

Dahari, O., \& De Robertis, M. M. 1988, ApJ, 331, 727

de Bruyn, A. G., \& Wilson, A. S. 1978, A\&A, 64, 433

de Vaucouleurs, G., de Vaucouleurs, A., Corwin, H. G., Jr., et al. 1991, Third Reference Catalogue of Bright Galaxies (New York, Berlin, Heidelberg: Springer-Verlag), 1

Edelson, R. A. 1987, ApJ, 313, 651

Falcke, H., Wilson, A. S., \& Simpson, C. 1998, ApJ, 502, 199

Falcke, H., Henkel, C., Peck, A. B., et al. 2000, A\&A, 358, L17

Ferruit, P., Wilson, A. S., \& Mulchaey, J. S. 1998, ApJ, 509, 646

Ferruit, P., Wilson, A. S., \& Mulchaey, J. 2000, ApJS, 128, 139

Gallimore, J. F., Axon, D. J., O’Dea, C. P., Baum, S. A., \& Pedlar, A. 2006, AJ, 132,546

Gallimore, J. F., Yzaguirre, A., Jakoboski, J., et al. 2010, ApJS, 187, 172

Genzel, R., Weitzel, L., Tacconi-Garman, L. E., et al. 1995, ApJ, 444, 129

Gonzalez Delgado, R. M., \& Perez, E. 1997, MNRAS, 284, 931

Gregory, P. C., \& Condon, J. J. 1991, ApJS, 75, 1011

Griffith, M. R., Wright, A. E., Burke, B. F., \& Ekers, R. D. 1995, ApJS, 97, 347

Heckman, T. M., Ptak, A., Hornschemeier, A., \& Kauffmann, G. 2005, ApJ, 634, 161

Ho, L. C., \& Ulvestad, J. S. 2001, ApJS, 133, 77

Kellermann, K. I., Sramek, R., Schmidt, M., Shaffer, D. B., \& Green, R. 1989, AJ, 98, 1195

Kewley, L. J., Groves, B., Kauffmann, G., \& Heckman, T. 2006, MNRAS, 372, 961

Khachikian, E. Y., \& Weedman, D. W. 1974, ApJ, 192, 581

Kharb, P., O'Dea, C. P., Baum, S. A., Colbert, E. J. M., \& Xu, C. 2006, ApJ, 652, 177

Kinney, A. L., Schmitt, H. R., Clarke, C. J., et al. 2000, ApJ, 537, 152

Kormendy, J., \& Gebhardt, K. 2001, in 20th Texas Symposium on relativistic astrophysics, eds. J. C. Wheeler, \& H. Martel, AIP Conf. Ser., 586, 363

Kukula, M. J., Pedlar, A., Baum, S. A., \& O'Dea, C. P. 1995, MNRAS, 276, 1262

Kukula, M. J., Dunlop, J. S., Hughes, D. H., \& Rawlings, S. 1998, MNRAS, 297, 366 
Lal, D. V., Shastri, P., \& Gabuzda, D. C. 2004, A\&A, 425, 99

Lal, D. V., Shastri, P., \& Gabuzda, D. C. 2011, ApJ, 731, 68

Lonsdale, C. J., Lonsdale, C. J., Smith, H. E., \& Diamond, P. J. 2003, ApJ, 592, 804

Maiolino, R., Ruiz, M., Rieke, G. H., \& Papadopoulos, P. 1997, ApJ, 485, 552

Malkan, M. A., Gorjian, V., \& Tam, R. 1998, ApJS, 117, 25

Mauder, W., Weigelt, G., Appenzeller, I., \& Wagner, S. J. 1994, A\&A, 285, 44

Mazzuca, L. M., Knapen, J. H., Veilleux, S., \& Regan, M. W. 2008, ApJS, 174, 337

McConnell, N. J., \& Ma, C.-P. 2013, ApJ, 764, 184

Middelberg, E., Roy, A. L., Nagar, N. M., et al. 2004, A\&A, 417, 925

Miyaji, T., Wilson, A. S., \& Perez-Fournon, I. 1992, ApJ, 385, 137

Momjian, E., Romney, J. D., Carilli, C. L., \& Troland, T. H. 2003, ApJ, 597, 809

Moran, E. C. 2000, New Astron. Rev., 44, 527

Moran, E. C., Barth, A. J., Kay, L. E., \& Filippenko, A. V. 2000, ApJ, 540, L73

Morganti, R., Tsvetanov, Z. I., Gallimore, J., \& Allen, M. G. 1999, A\&AS, 137, 457

Mulchaey, J. S., Wilson, A. S., \& Tsvetanov, Z. 1996, ApJ, 467, 197

Mundell, C. G., Holloway, A. J., Pedlar, A., et al. 1995, MNRAS, 275, 67

Mundell, C. G., Wilson, A. S., Ulvestad, J. S., \& Roy, A. L. 2000, ApJ, 529, 816

Mundell, C. G., Wrobel, J. M., Pedlar, A., \& Gallimore, J. F. 2003, ApJ, 583, 192

Mundell, C. G., Ferruit, P., Nagar, N., \& Wilson, A. S. 2009, ApJ, 703, 802

Nagar, N. M., \& Wilson, A. S. 1999, ApJ, 516, 97

Nagar, N. M., Wilson, A. S., Mulchaey, J. S., \& Gallimore, J. F. 1999, ApJS, 120, 209

Neff, S. G., \& de Bruyn, A. G. 1983, A\&A, 128, 318

Nelson, C. H., \& Whittle, M. 1995, ApJS, 99, 67

Panessa, F., \& Bassani, L. 2002, A\&A, 394, 435

Pedlar, A., Kukula, M. J., Longley, D. P. T., et al. 1993, MNRAS, 263, 471

Polletta, M., Bassani, L., Malaguti, G., Palumbo, G. G. C., \& Caroli, E. 1996, ApJS, 106, 399

Pringle, J. E., Antonucci, R. R. J., Clarke, C. J., et al. 1999, ApJ, 526, L9

Rush, B., Malkan, M. A., \& Edelson, R. A. 1996, ApJ, 473, 130

Schmidt, M., \& Green, R. F. 1983, ApJ, 269, 352
Schmitt, H. R., Antonucci, R. R. J., Ulvestad, J. S., et al. 2001, ApJ, 555, 663 Schmitt, H. R., Donley, J. L., Antonucci, R. R. J., Hutchings, J. B., \& Kinney, A. L. 2003a, ApJS, 148, 327

Schmitt, H. R., Donley, J. L., Antonucci, R. R. J., et al. 2003b, ApJ, 597, 768

Schommer, R. A., Caldwell, N., Wilson, A. S., et al. 1988, ApJ, 324, 154

Shastri, P., Lal, D. V., \& Gabuzda, D. C. 2003, in Active Galactic Nuclei: From Central Engine to Host Galaxy, eds. S. Collin, F. Combes, \& I. Shlosman, ASP Conf. Ser., 290, 311

Singh, V., Shastri, P., \& Risaliti, G. 2011, A\&A, 532, A84

Swarup, G., Ananthakrishnan, S., Kapahi, V. K., et al. 1991, Current science, 60, 95

Thean, A., Pedlar, A., Kukula, M. J., Baum, S. A., \& O’Dea, C. P. 2000, MNRAS, 314, 573

Thean, A., Pedlar, A., Kukula, M. J., Baum, S. A., \& O’Dea, C. P. 2001 , MNRAS, 325, 737

Tran, H. D. 2001, ApJ, 554, L19

Tran, H. D. 2003, ApJ, 583, 632

Ulvestad, J. S., \& Ho, L. C. 2001, ApJ, 558, 561

Ulvestad, J. S., \& Wilson, A. S. 1984a, ApJ, 278, 544

Ulvestad, J. S., \& Wilson, A. S. 1984b, ApJ, 285, 439

Ulvestad, J. S., \& Wilson, A. S. 1989, ApJ, 343, 659

Ulvestad, J. S., Wilson, A. S., \& Sramek, R. A. 1981, ApJ, 247, 419

Ulvestad, J. S., Wrobel, J. M., Roy, A. L., et al. 1999, ApJ, 517, L81

Ulvestad, J. S., Wong, D. S., Taylor, G. B., Gallimore, J. F., \& Mundell, C. G. 2005, AJ, 130, 936

Unger, S. W., Pedlar, A., Axon, D. J., et al. 1988, MNRAS, 234, 745

Urry, K., Padovanni, G., Wilson, A. S., \& Yoshida, M. 1995, ApJ, 521, 565

Wang, J., Mao, Y. F., \& Wei, J. Y. 2009, AJ, 137, 3388

Weedman, D. W. 1977, ARA\&A, 15, 69

White, R. L., \& Becker, R. H. 1992, ApJS, 79, 331

Whittle, M. 1992, ApJS, 79, 49

Wilson, A. S., \& Tsvetanov, Z. I. 1994, AJ, 107, 1227

Wilson, A. S., \& Ulvestad, J. S. 1982a, ApJ, 260, 56

Wilson, A. S., \& Ulvestad, J. S. 1982b, ApJ, 263, 576

Wilson, A. S., Helfer, T. T., Haniff, C. A., \& Ward, M. J. 1991, ApJ, 381, 79

Wrobel, J. M. 2000, ApJ, 531, 716 
A\&A 554, A85 (2013)
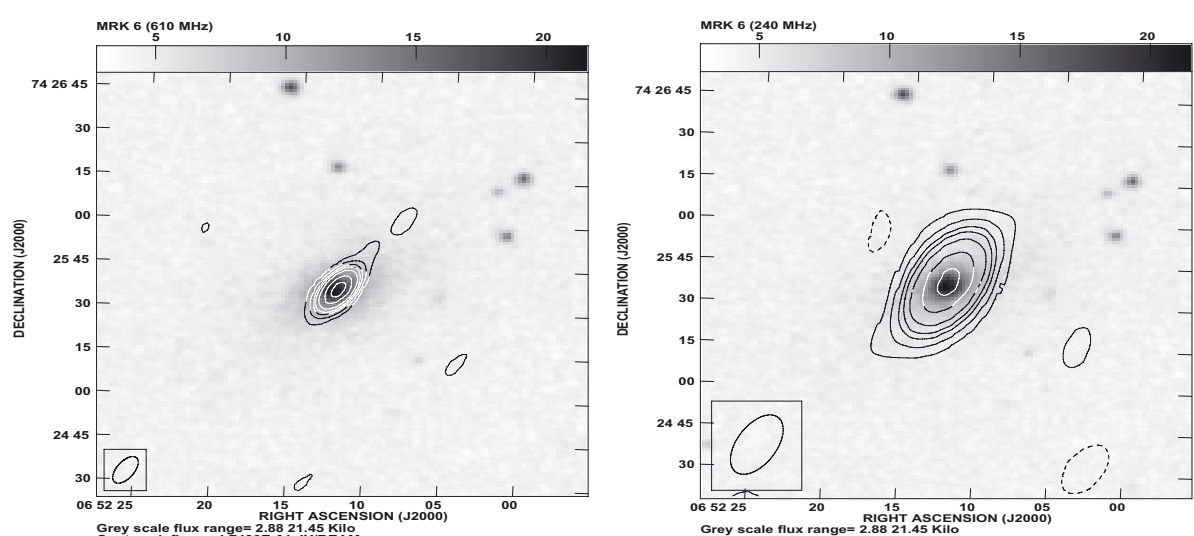

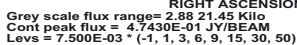
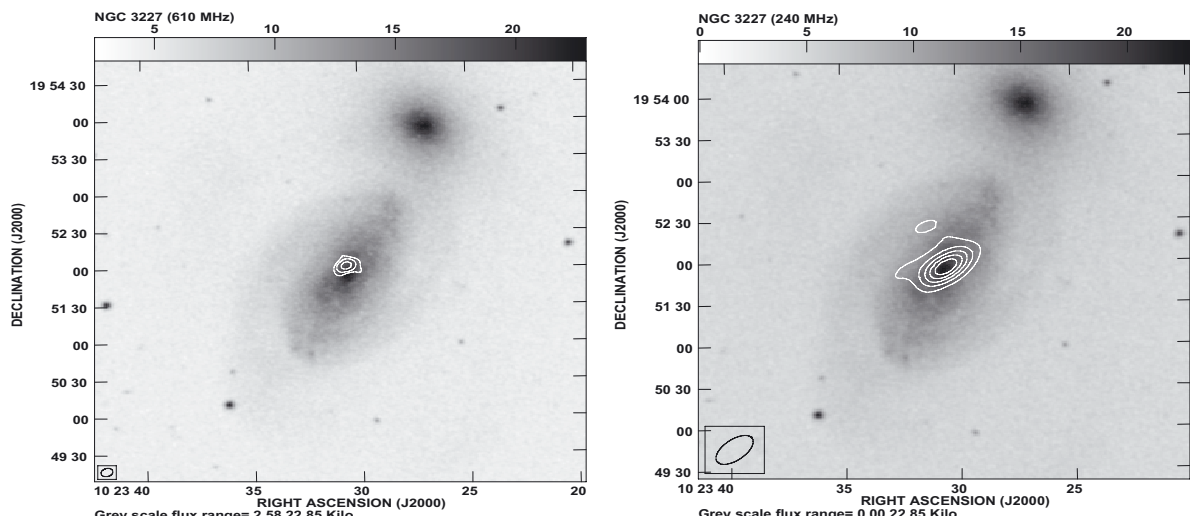

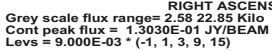
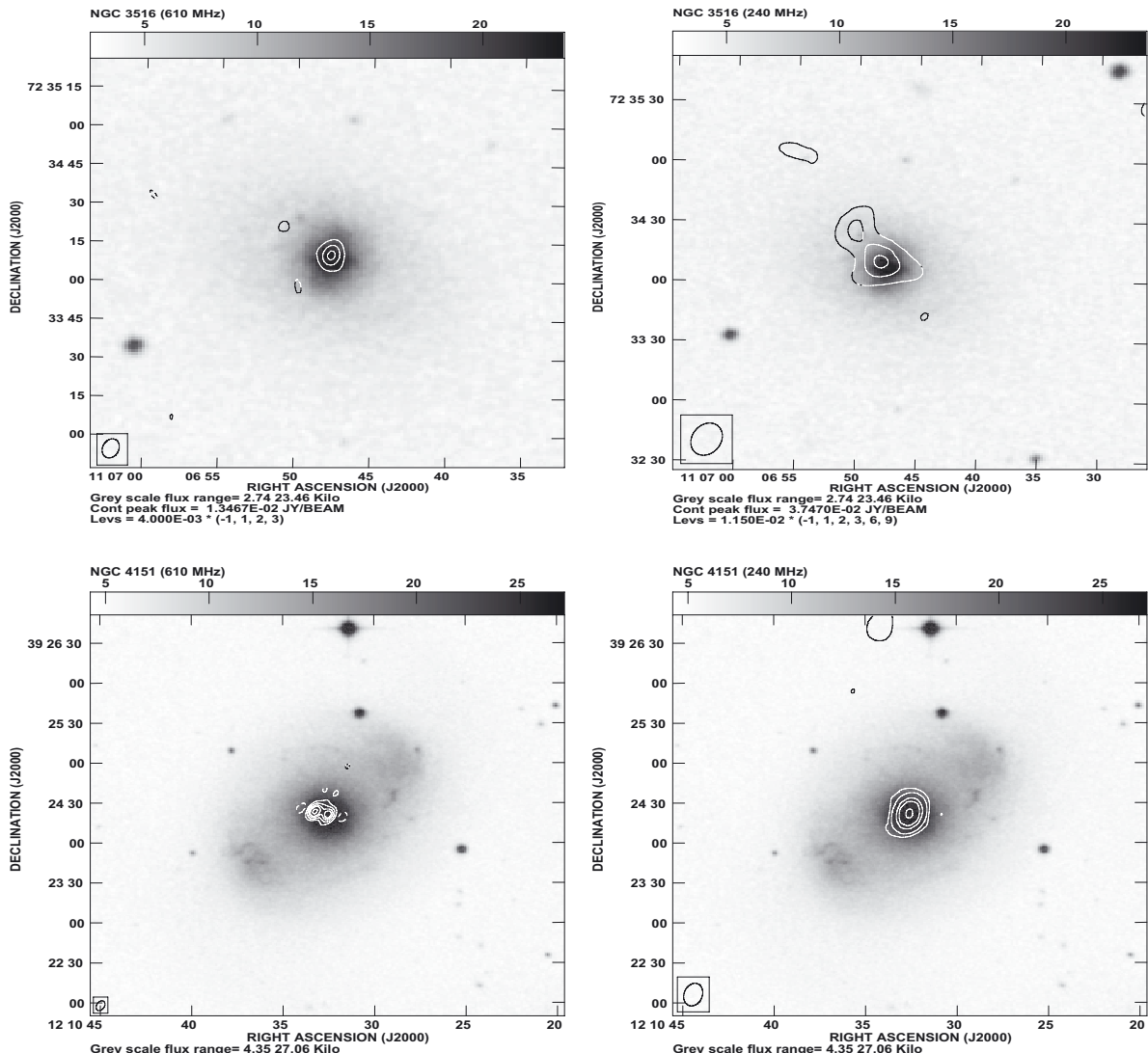

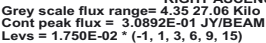

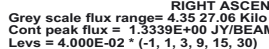

Fig. 2. $610 \mathrm{MHz}$ (left panel) and $240 \mathrm{MHz}$ (right panel) radio contours overlaid on their DSS optical images. The restoring beam is shown in lower left corner of each map. The contour levels are shown at the bottom of each map. The first lowest radio contour is about $4 \sigma-5 \sigma$ of the rms noise value in each map. The source name and radio frequency is mentioned at the top left of each map. The same plotting convention is followed for other sources. 
V. Singh et al.: Low-frequency radio observations of Seyfert galaxies: A test of the unification scheme
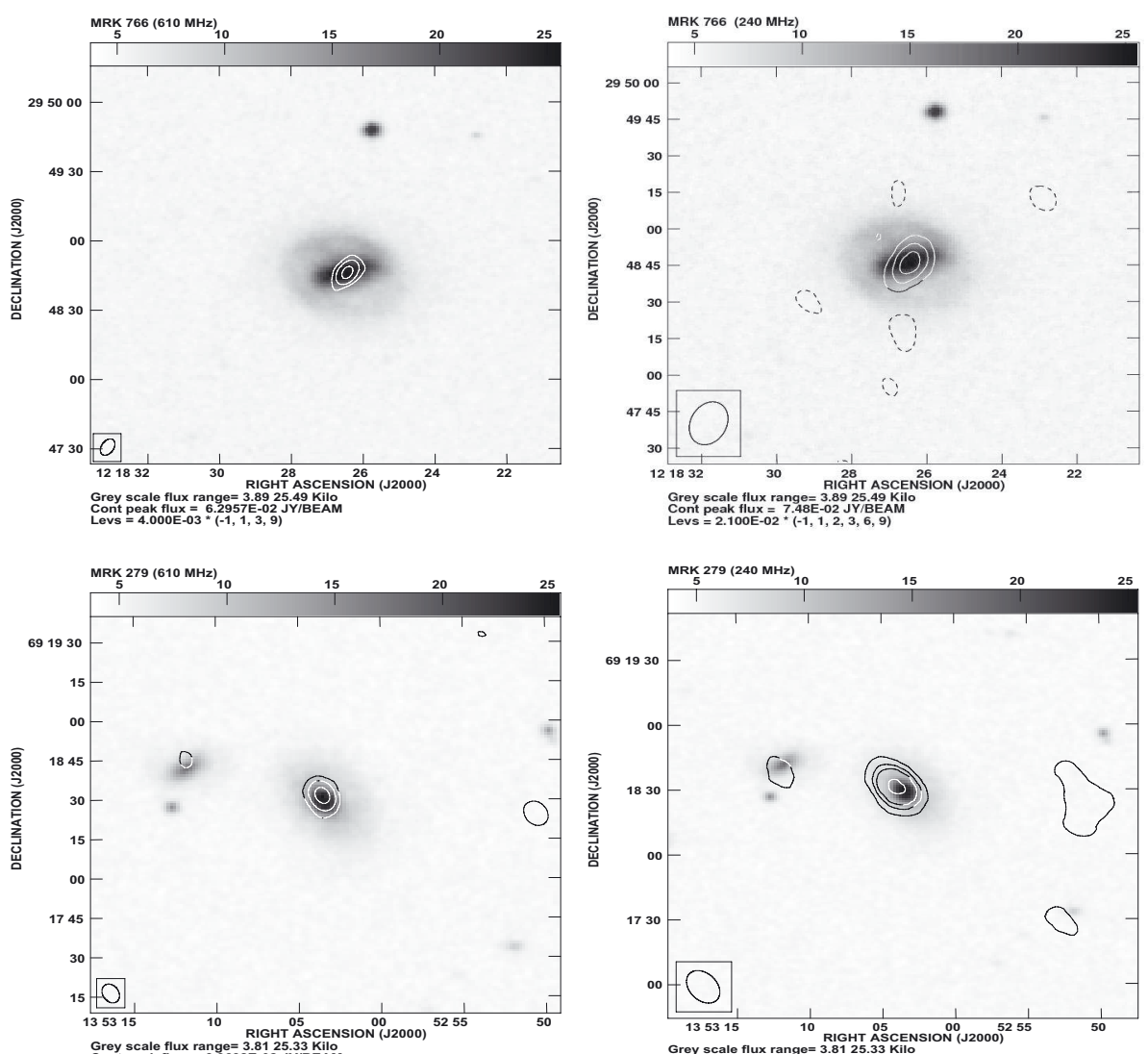

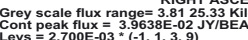
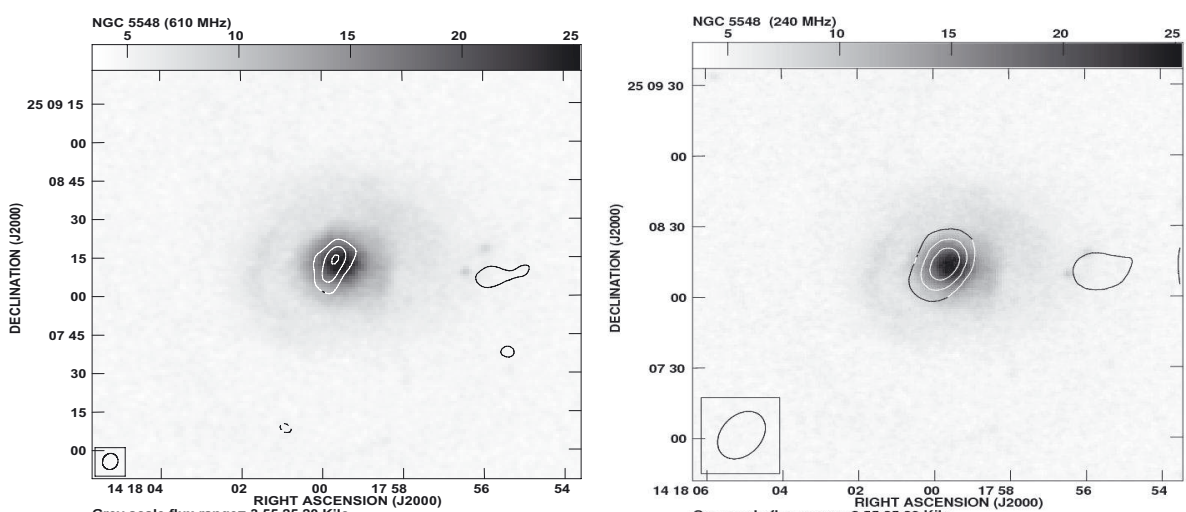

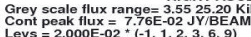
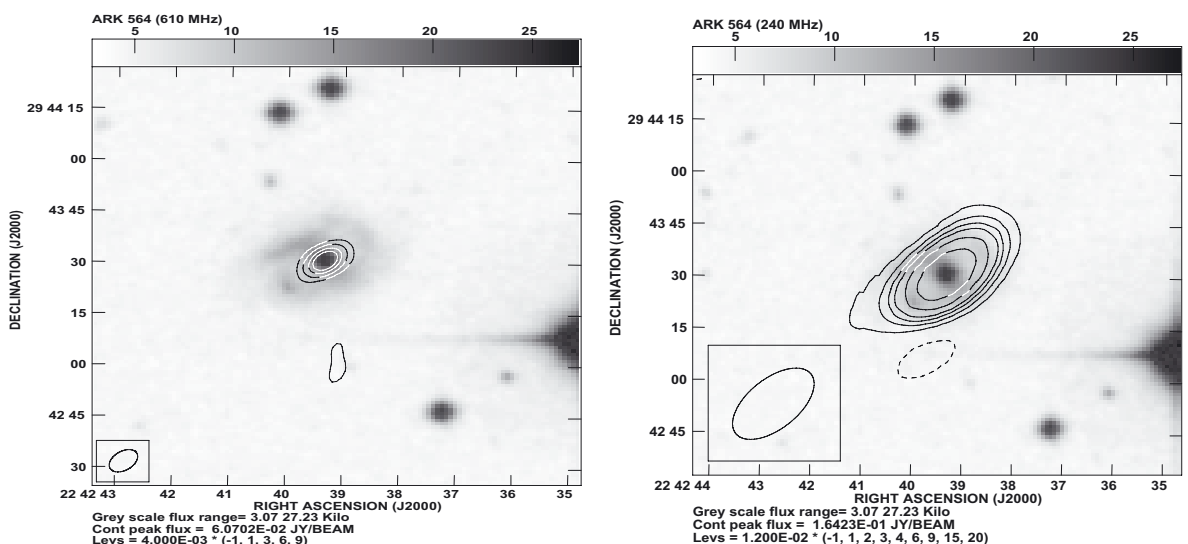

Fig. 2. continued. 
A\&A 554, A85 (2013)
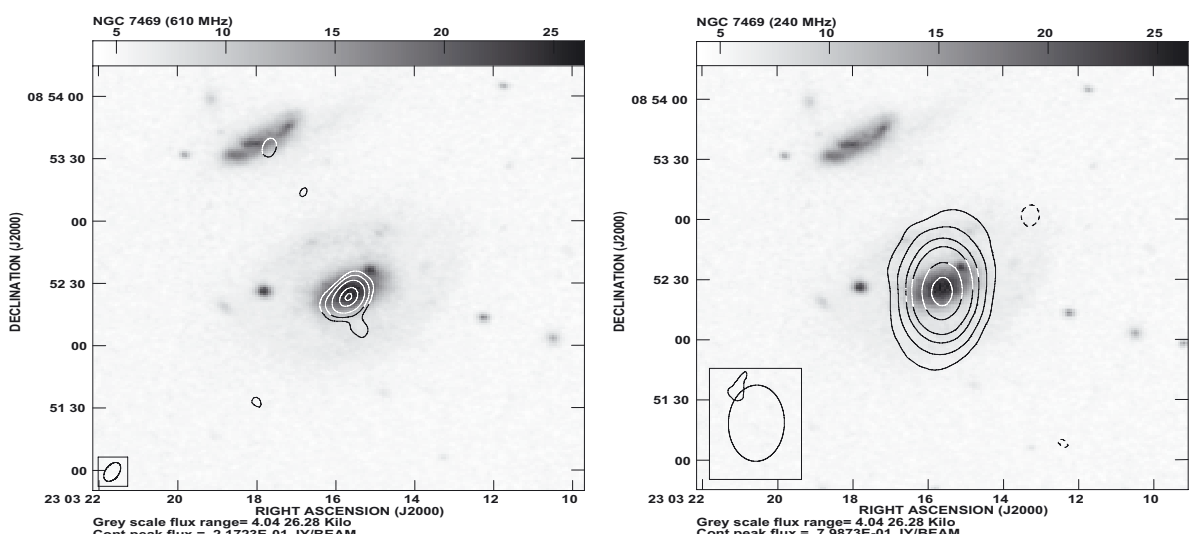

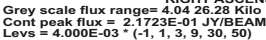
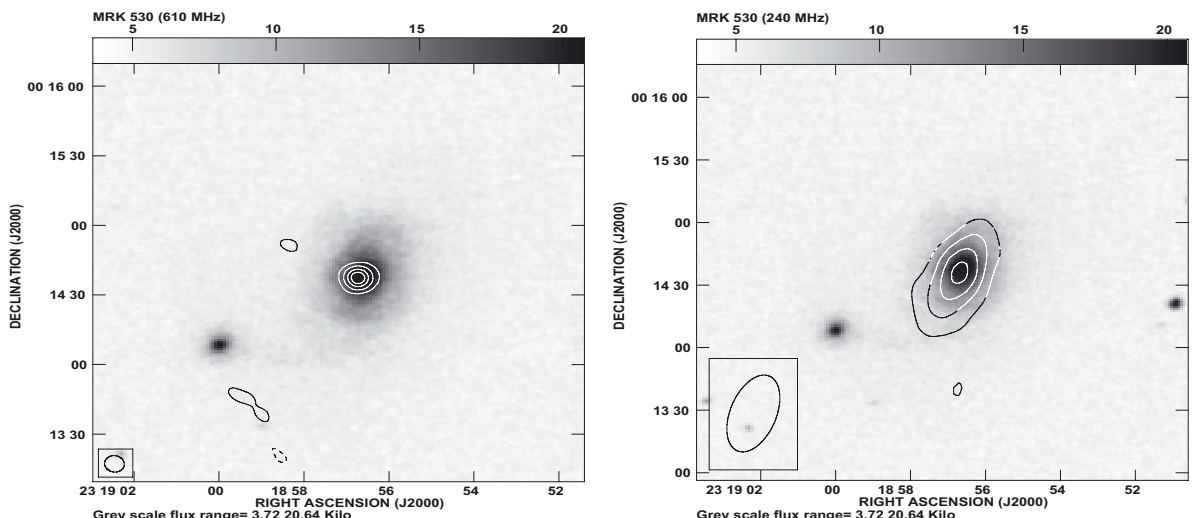

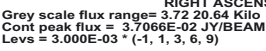
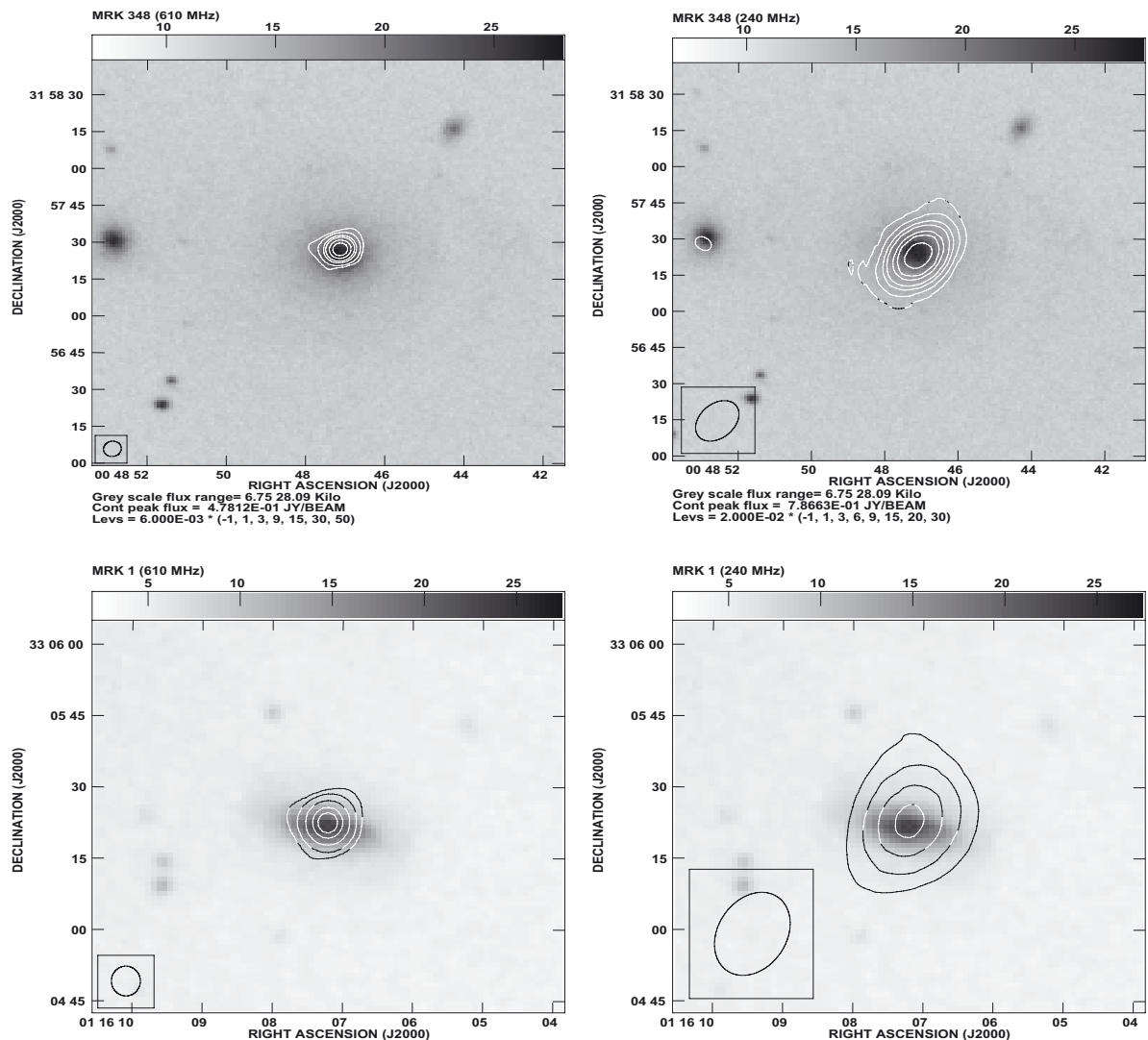

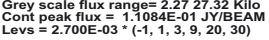

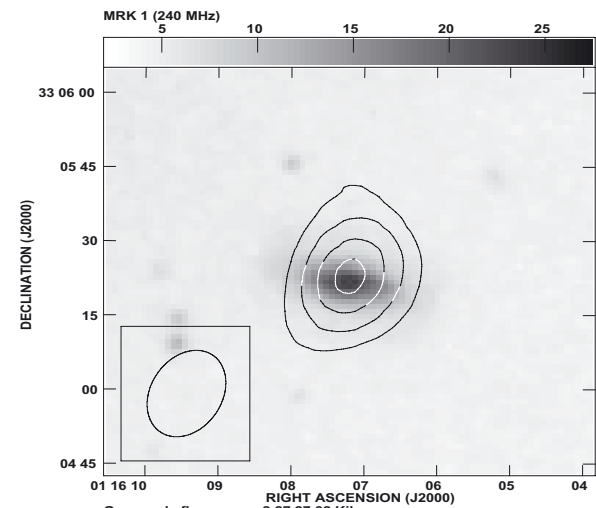

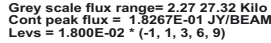

Fig. 2. continued. 
V. Singh et al.: Low-frequency radio observations of Seyfert galaxies: A test of the unification scheme
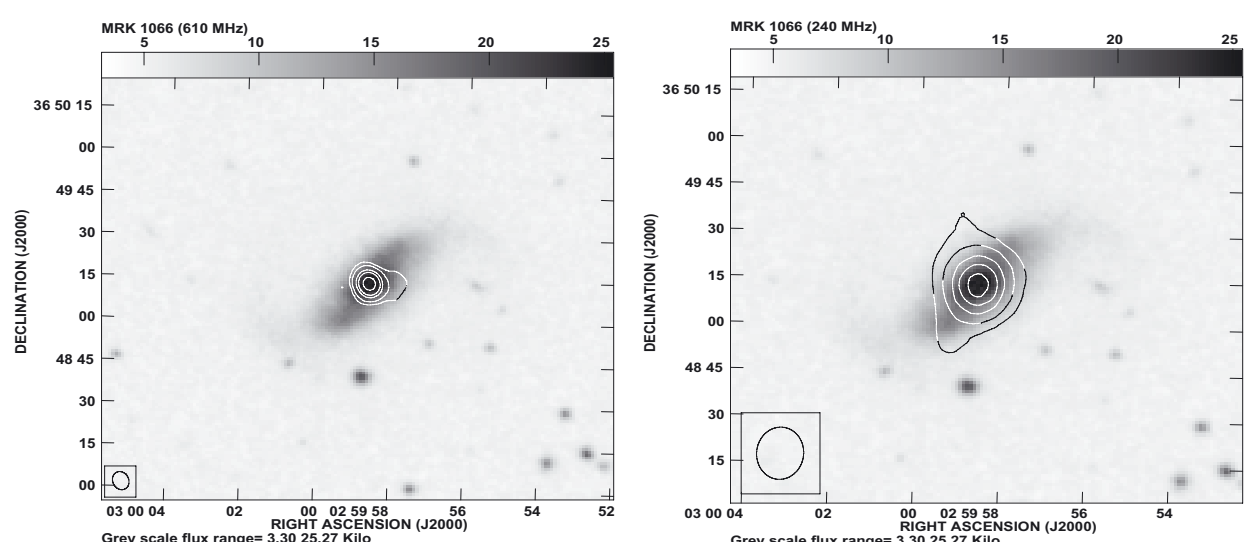

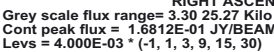
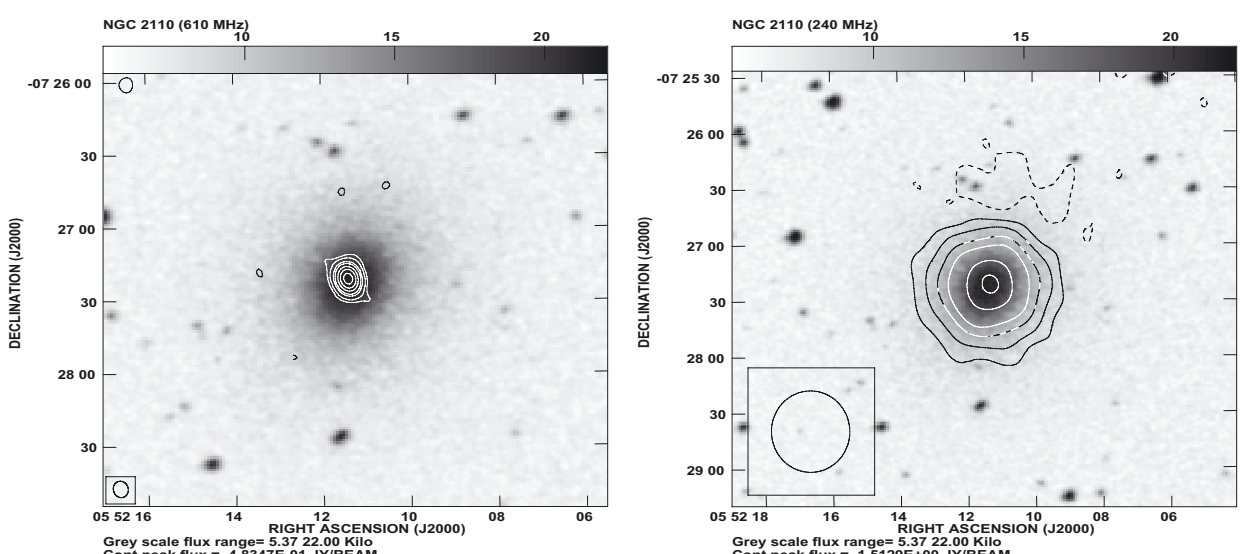

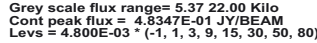
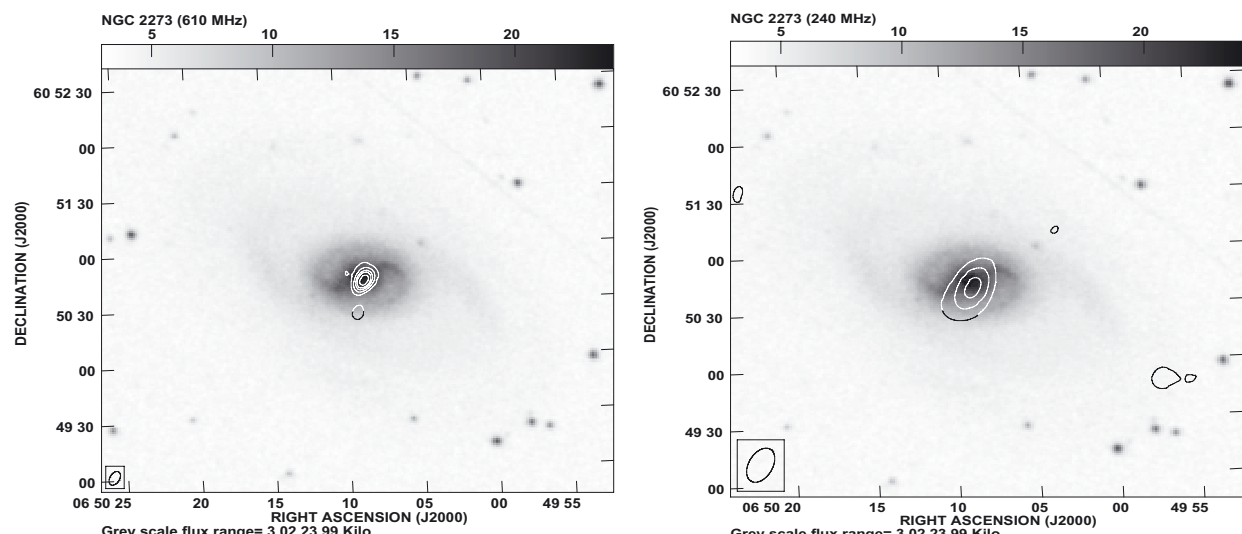

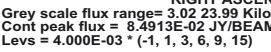

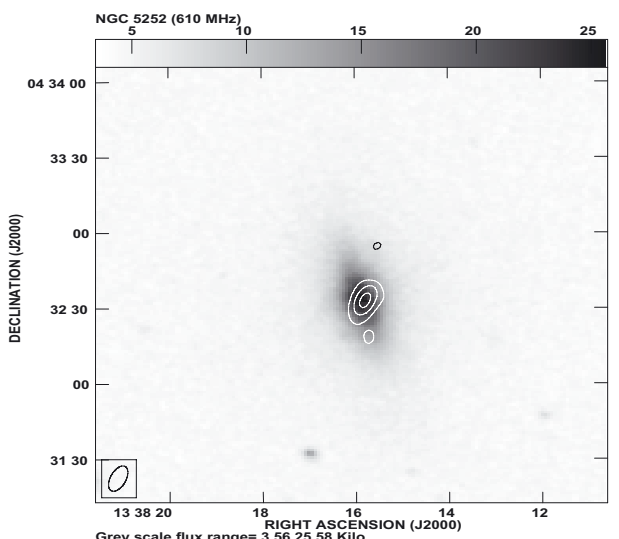

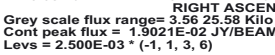

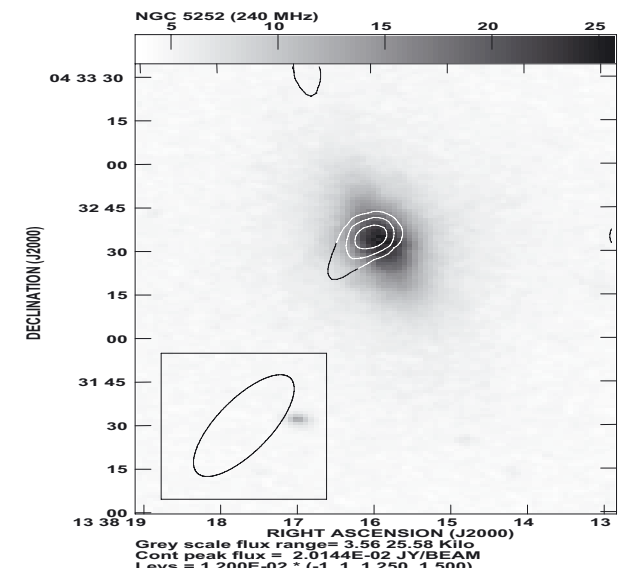

Fig. 2. continued. 
A\&A 554, A85 (2013)
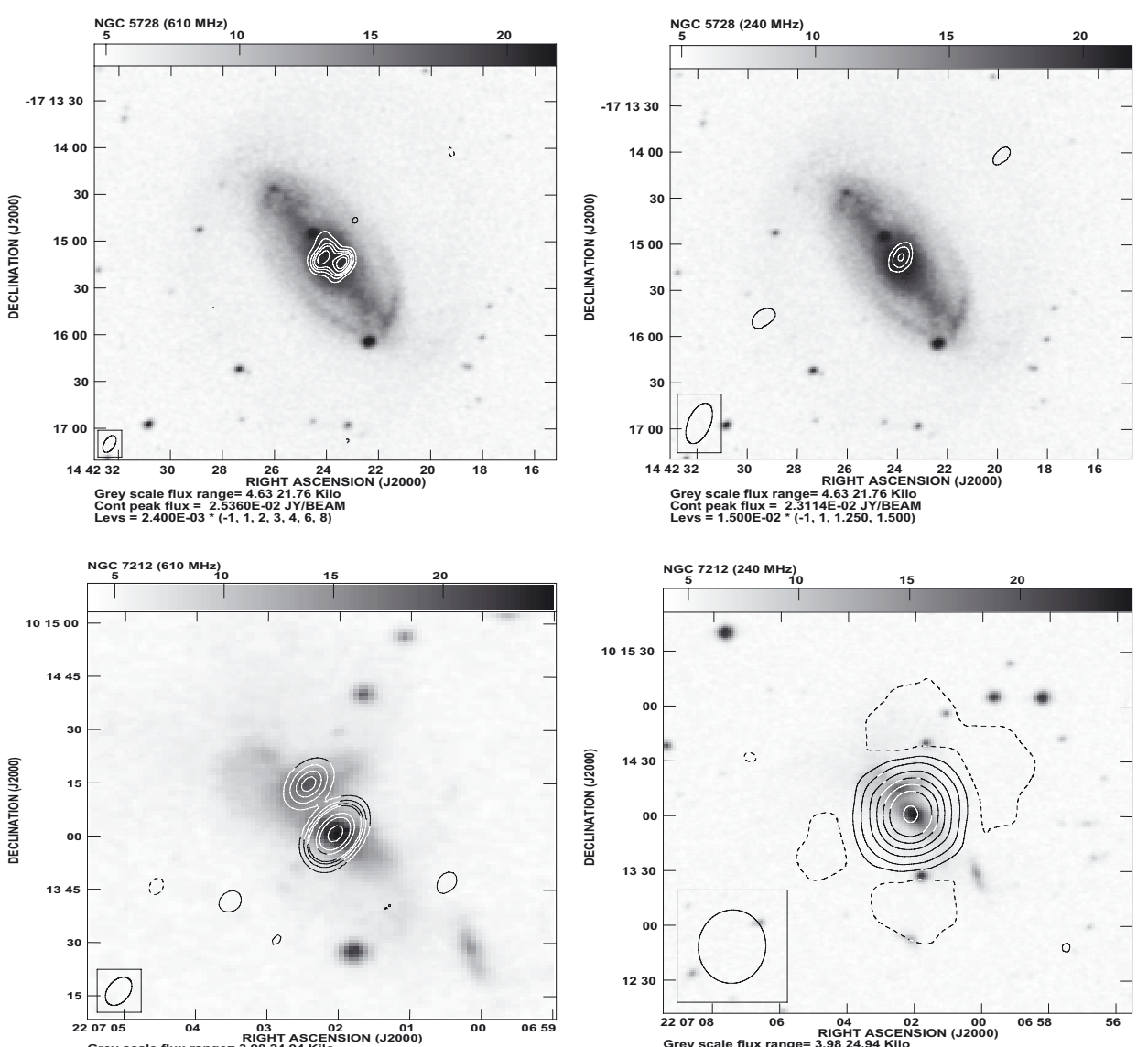

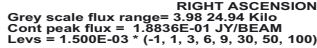
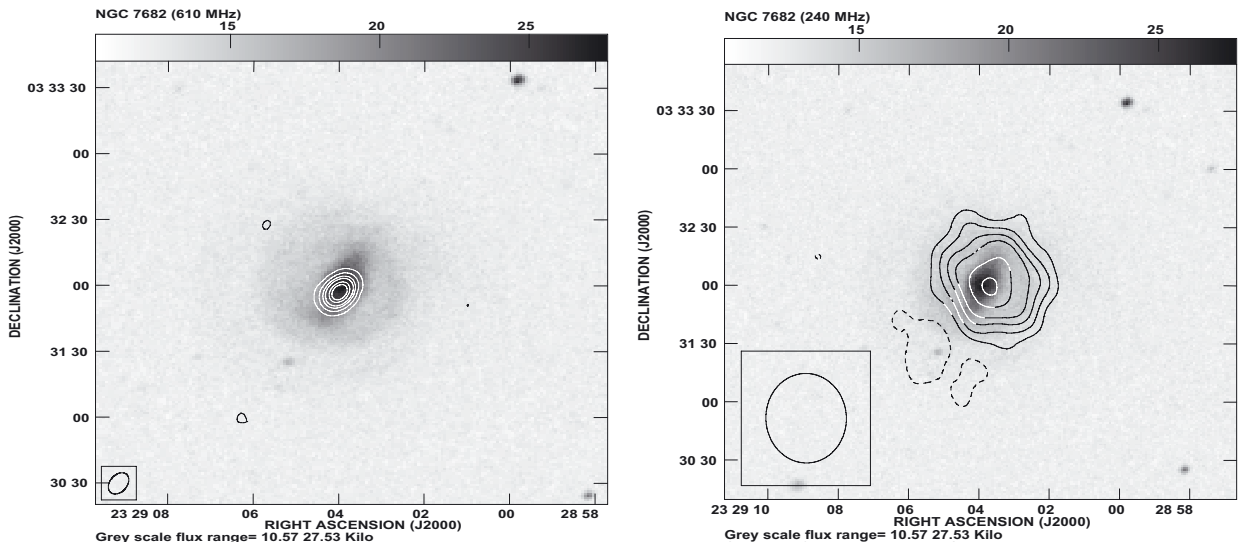

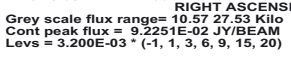
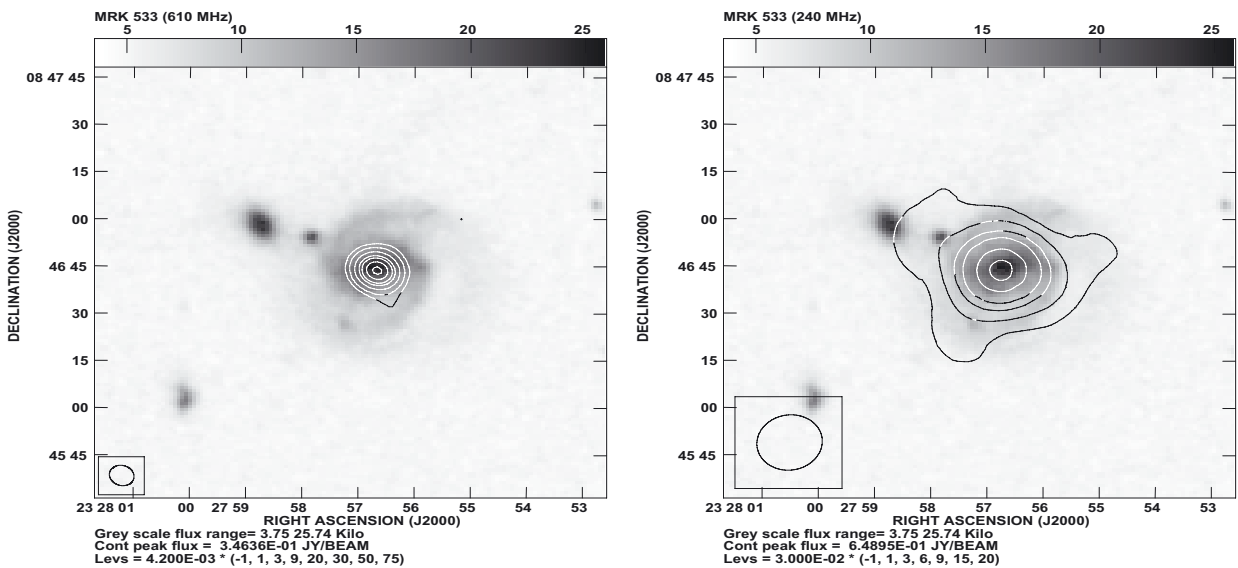

Fig. 2. continued. 
V. Singh et al.: Low-frequency radio observations of Seyfert galaxies: A test of the unification scheme
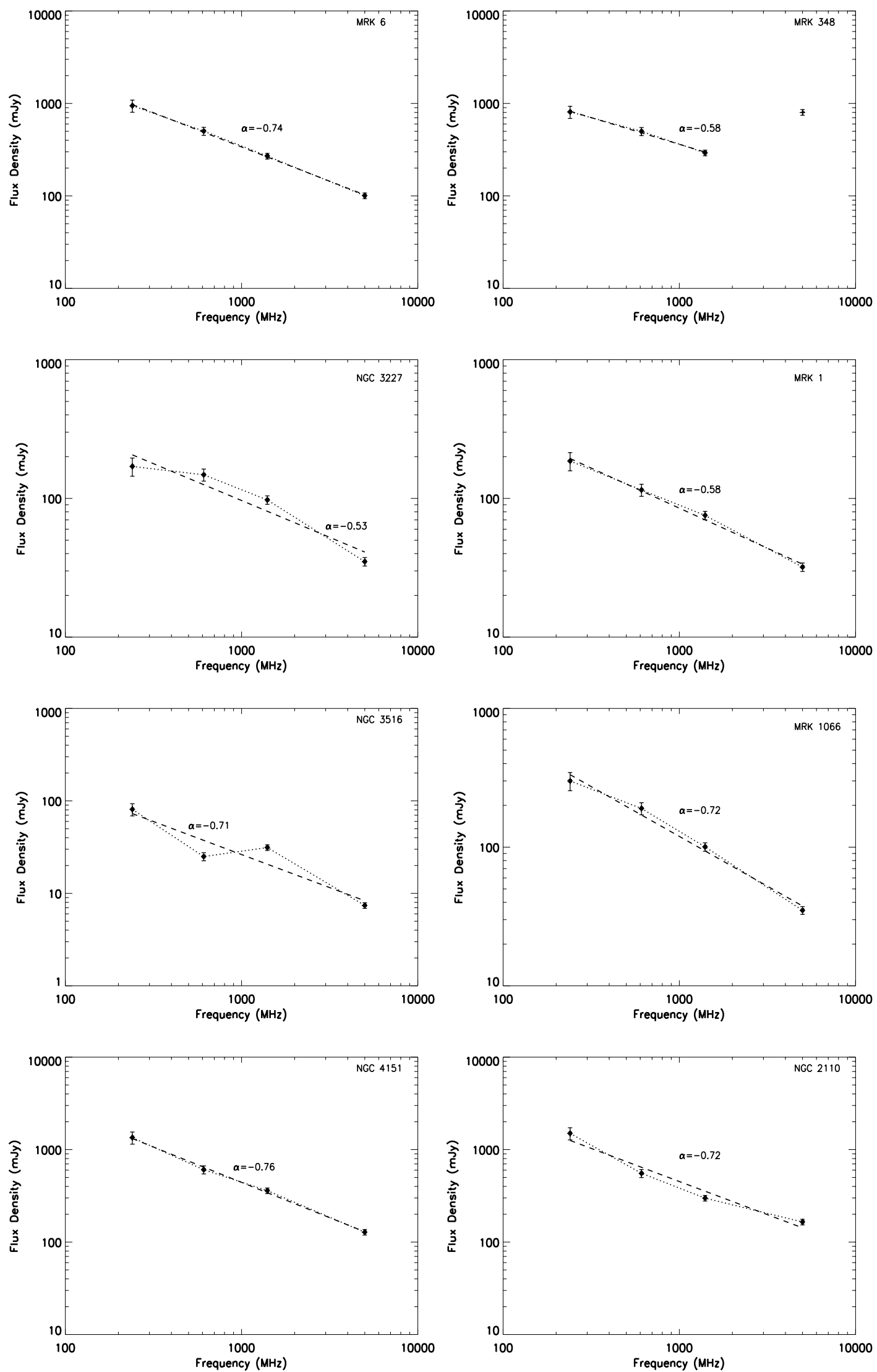

Fig. 4. Four-point (240 MHz, $610 \mathrm{MHz}, 1.4 \mathrm{GHz}$, and $5.0 \mathrm{GHz}$ ) radio spectra of Seyfert galaxies in our sample. The dotted line connects the flux density points and dashed line shows the least chi-square linear fit to the spectral points. Radio spectra of Seyfert types $1 \mathrm{~s}$ and $2 \mathrm{~s}$ are shown in the left and right panels, respectively. 
A\&A 554, A85 (2013)
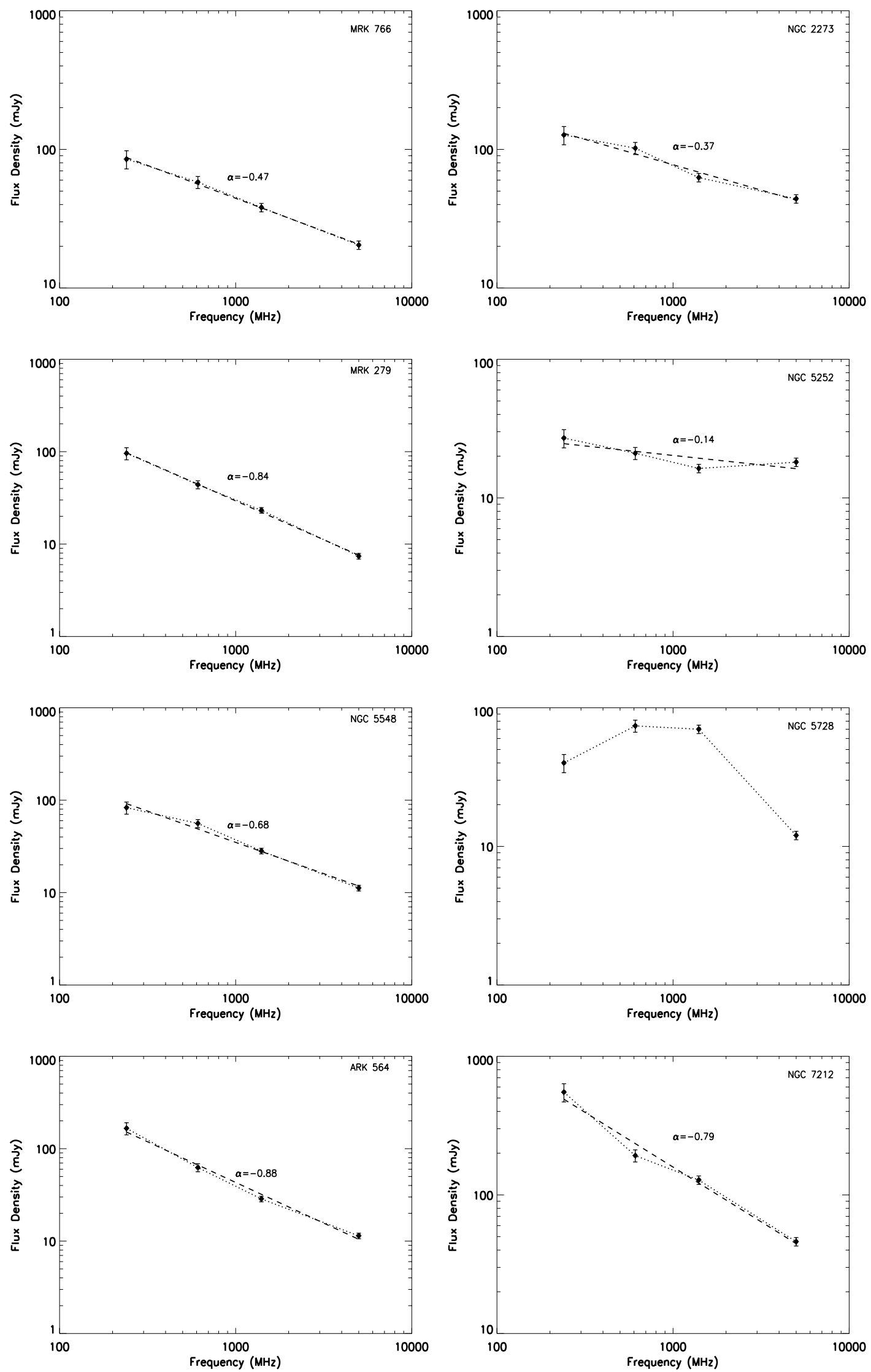

Fig. 4. continued. 
V. Singh et al.: Low-frequency radio observations of Seyfert galaxies: A test of the unification scheme
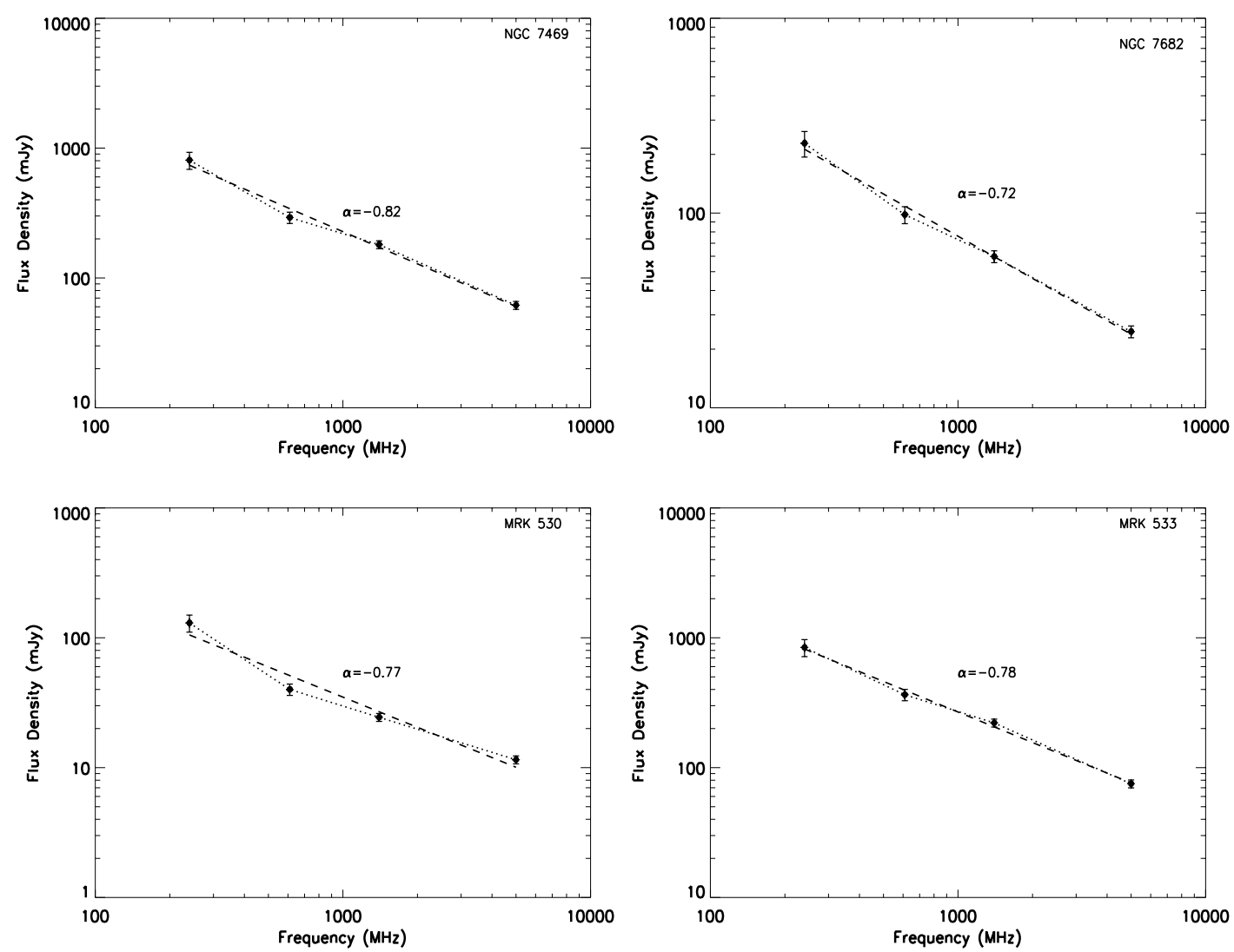

Fig. 4. continued. 


\section{Appendix A: Notes on individual sources}

In this section we describe the radio properties of the individual sources in our sample.

\section{A.1. MRK 6}

Kharb et al. (2006) present a detailed study of MRK 6 radio morphology and report that the radio emission is seen on three different spatial scales i.e., $\sim 7.5 \mathrm{kpc}$ bubbles, $\sim 1.5 \mathrm{kpc}$ bubbles lying nearly orthogonal to each other, and a $\sim 1 \mathrm{kpc}$ radio jet lying orthogonal to the $1.5 \mathrm{kpc}$ bubble. They suggest that the existence of $\mathrm{kpc}$ scale radio bubbles/lobes can be attributed to the radio relics related to past episodic nuclear activity. Our radio images at $610 \mathrm{MHz}$ and $240 \mathrm{MHz}$ show only a single component that is fitted with elliptical Gaussians of 10 ' $42 \times 55^{\prime \prime} 06$ and 24.' $14 \times 11^{\prime \prime}$.76, respectively. The radio emission at $610 \mathrm{MHz}$ and $240 \mathrm{MHz}$ are likely to include all the emitting components detected by Kharb et al. (2006) because the combined $5.0 \mathrm{GHz}$ flux density of all the components in Kharb et al. (2006) follow the spectral shape determined by our $240 \mathrm{MHz}, 610 \mathrm{MHz}$ GMRT, and 1.4 GHz NVSS flux densities.

\section{A.2. NGC 3227}

The 8.4 GHz VLA observations show north-south elongated nuclear radio emission, and at sub-arcsec resolution, the core is resolved into 0.'4 double sources (Mundell et al. 1995). The 5.0 GHz VLA "D" array observations show the kpc-scale extended radio emission, which is interpreted as the emission from star-forming host galaxy disk (Gallimore et al. 2006). Our GMRT images at $610 \mathrm{MHz}$ and $240 \mathrm{MHz}$ show radio emission as nearly unresolved point sources that are fitted with an elliptical Gaussian of 8.' $85 \times 66^{\prime \prime} 70$ and 32 .' $61 \times 18^{\prime \prime} .02$, respectively. The fitted powerlaw radio spectrum over $240 \mathrm{MHz}$ to $5.0 \mathrm{GHz}$ renders spectral index $\left(\alpha_{\text {int }}\right) \sim-0.53$, while there is an indication of spectral flattening toward lower frequencies.

\section{A.3. NGC 3516}

High-resolution VLA observations of NGC 3516 show a compact flat-spectrum core with a $\sim 0$ ' $7(\sim 120$ pc) one-sided northern extension along $\mathrm{PA} \simeq 8^{\circ}$ (Nagar et al. 1999). At arcsec resolution, additional radio components are seen that extend out to $\sim 4^{\prime \prime}(\sim 740 \mathrm{pc})$ and align with the co-spatial S-shaped optical emission-line structure (Miyaji et al. 1992; Ferruit et al. 1998). At coarser resolution, a linear radio structure spanning $\sim 45^{\prime \prime}(\sim 8.3 \mathrm{kpc})$, oriented along PA $\sim 44^{\circ}$, across the nucleus is also seen (Baum et al. 1993). In our GMRT observations, the $610 \mathrm{MHz}$ radio emission is detected as marginally resolved point source fitted with an elliptical Gaussian of 10 '. $44 \times 77^{\prime \prime} .38$. The integrated flux density at $610 \mathrm{MHz}$ is substantially higher than the peak flux density, and also the fitted Gaussian size is larger than the synthesized beam size, and this may be indicative of the presence of extended emission at the kpc-scale. We fit the $240 \mathrm{MHz}$ radio emission with a large Gaussian of $377^{\prime \prime} 09 \times 144^{\prime \prime} 31$. There is hint of second component along the north-east direction from the central nuclear component that seems to be consistent with the radio morphology reported by Baum et al. (1993). NGC 3516 shows inverted spectrum between $1.4 \mathrm{GHz}$ and $610 \mathrm{MHz}$, while a steep spectrum between $610 \mathrm{MHz}$ and $240 \mathrm{MHz}$. The changing spectral behavior can be interpreted as the presence of at least two emitting components. Possibly, $5.0 \mathrm{GHz}$ to $610 \mathrm{MHz}$ is dominated by the compact AGN core having self absorbed synchrotron emission, and low-frequency emission at $610 \mathrm{MHz}$ to $240 \mathrm{MHz}$ is dominated by optically thin synchrotron emission from the extended component.

\section{A.4. NGC 4151}

The nuclear radio source in NGC 4151 resolves into compact knots aligned in a slightly curved jet structure spanning $\sim 5^{\prime \prime}$ $\left(\sim 340\right.$ parsec) across the nucleus and oriented along PA $\sim 80^{\circ}$ (Ulvestad et al. 1981; Booler et al. 1982; Wilson \& Ulvestad 1982b; Pedlar et al. 1993; Mundell et al. 2003). The WSRT observations of NGC 4151 revealed a $\sim 15^{\prime \prime}(\sim 1 \mathrm{kpc})$ linear structure, roughly aligned with the inner jet, and a bracketing pair of radio continuum arcs located at $\sim 45^{\prime \prime}(\sim 3 \mathrm{kpc})$ from the radio nucleus (Baum et al. 1993). In our GMRT observations, the $610 \mathrm{MHz}$ radio emission is double-peaked with the weaker peak at a distance of $\sim 8^{\prime \prime} .54$ along the PA $\sim 65^{\circ} .2$ from the central component. The $610 \mathrm{MHz}$ radio emission can be fitted with two elliptical Gaussian components with the central component having a peak flux density $\sim 312.9 \mathrm{mJy} /$ beam and convolved size $\sim 8^{\prime \prime} .12 \times 55^{\prime \prime}$. 28 , while the second Gaussian component has peak flux density of $\sim 218.1 \mathrm{mJy} /$ beam and convolved size $\sim 8^{\prime \prime}$. $30 \times 5$ ". 74. Owing to its larger beam-size, the $240 \mathrm{MHz}$ radio image shows a nearly unresolved point source fitted with a Gaussian of $\sim 17$ '. $10 \times 11^{\prime \prime}$. 64 . The four-point radio spectrum remains fairly steep $\left(\alpha_{\text {int }} \sim-0.76\right)$ over $240 \mathrm{MHz}$ to $5.0 \mathrm{GHz}$.

\section{A.5. MRK 766}

The 8.4 GHz VLA “A” and "C" array observations of MRK 766 show an unresolved point source emission and no evidence of emission on intermediate scale (Kukula et al. 1995). In our GMRT observations, the $610 \mathrm{MHz}$ and $240 \mathrm{MHz}$ radio emission is seen as unresolved point sources that are fitted with Gaussian of $\sim 7$ '. $^{\prime} 92 \times 4$ 4. $^{\prime} 81$ and $\sim 16^{\prime \prime} .20 \times 9$ '. 90 , respectively. The fourpoint integrated radio spectral index $\left(\alpha_{\text {int }} \sim-0.47\right)$ is less steep than average spectral index for our sample Seyfert galaxies.

\section{A.6. MRK 279}

VLA observations of MRK 279 at $8.4 \mathrm{GHz}$ show an unresolved point source emission in both " $\mathrm{A}$ " and " $\mathrm{C}$ " array configurations and no evidence of emission on intermediate scale (Kukula et al. 1995). Our GMRT observations at $610 \mathrm{MHz}$ and $240 \mathrm{MHz}$ show radio emission as an unresolved point source fitted with the Gaussians of sizes $\sim 7$ ". $^{\prime} 86 \times 5$ ". 54 and $\sim 17^{\prime \prime}$. $69 \times 11^{\prime \prime}$. 52 , respectively. The four-point radio spectrum of MRK 279 is fairly steep with an integrated spectral index $\left(\alpha_{\text {int }}\right) \sim-0.88$.

\section{A.7. NGC 5548}

VLA observations of NGC 5548 revealed a compact core between two diffuse lobes separated by $\sim 15^{\prime \prime}$ (Wilson \& Ulvestad 1982a). Using VLA “D” array observations, Gallimore et al. (2006) detected marginally resolved lobes along with the nuclear point source emission. In our GMRT observations, we fit the $610 \mathrm{MHz}$ radio emission with a Gaussian of $\sim 144^{\prime \prime} 33 \times 77^{\prime \prime} 11$. We note that the $610 \mathrm{MHz}$ total flux density is significantly higher than the peak flux density, and also the fitted Gaussian size is larger than the synthesized beam size. This may infer the presence of kpc-scale extended radio emission. At $240 \mathrm{MHz}$, the radio emission is nearly an unresolved point source fitted with a 
Gaussian of $\sim 23$ '.54 $\times 14$ ". 83 . The four-point radio spectrum of NGC 5548 is fitted with a spectral index of $\left(\alpha_{\text {int }}\right) \sim-0.68$.

\section{A.8. ARK 564}

The $8.4 \mathrm{GHz}$ VLA observations of ARK 564 show a triple radio source along the north-south direction $\left(\mathrm{PA} \sim 6^{\circ}\right.$ ), extended to $\sim 320 \mathrm{pc}$ (Moran 2000). In our GMRT observations, the radio emission at $610 \mathrm{MHz}$ and $240 \mathrm{MHz}$ is nearly pointsource emission fitted with a Gaussian of 7.'62 $\times 4$ ".'91 and $\sim 24$ ". $29 \times 12^{\prime \prime}$.92, respectively. The four-point radio spectrum over $240 \mathrm{MHz}$ to $5.0 \mathrm{GHz}$ is fairly steep with an integrated spectral index $\left(\alpha_{\text {int }}\right) \sim-0.88$.

\section{A.9. NGC 7469}

The 1.6 GHz VLBI observations resolved the core-jet structure into five different components, lying in an east-west line which extends up to $\sim 55 \mathrm{pc}$ (Lonsdale et al. 2003). A variety of observations at high spatial resolution indicate that the AGN in NGC 7469 is surrounded by a ring of starburst (Mauder et al. 1994; Genzel et al. 1995) with radio observations showing an unresolved central component surrounded by the ring-like structure (Wilson et al. 1991). In our GMRT observations, the radio emission at $610 \mathrm{MHz}$ and 240 is fitted with single Gaussian components with sizes of $\sim 10$ !' $43 \times 66^{\prime \prime} 70$ and $\sim 37^{\prime \prime} .86 \times 20$ ' $^{\prime} 68$, respectively. The integrated flux density at $610 \mathrm{MHz}$ is substantially higher than the peak flux density, and the fitted Gaussian size is also larger than the synthesized beam size. This may be considered as an indication of kpc-scale extended emission in NGC 7469. The four-point radio spectrum of NGC 7469 is fairly steep with integrated spectral index $\left(\alpha_{\text {int }}\right) \sim-0.82$.

\section{A.10. MRK 530}

VLA observations of MRK 530 at $8.4 \mathrm{GHz}$ show a compact point source emission with a slight extension (Kukula et al. 1995). The milli-arcsec resolution VLBI observations show a compact core emission with extension along the east-west direction at fainter levels (Lal et al. 2004). In our GMRT observations, the radio emission at $610 \mathrm{MHz}$ and $240 \mathrm{MHz}$ is seen as nearly point source that is fitted with single Gaussian components of sizes $\sim 7$ '. $^{\prime} 8 \times 7^{\prime \prime}$. 18 and $\sim 466^{\prime} .92 \times 21^{\prime \prime}$. 46 , respectively. The fourpoint radio spectrum of MRK 530 is fairly steep $\left(\alpha_{\text {int }}\right) \sim-0.77$.

\section{A.11. $M R K 348$}

In MRK 348, the radio continuum emission is dominated by a variable, subparsec-scale $(\sim 0.5 \mathrm{pc})$ jet that feeds into a larger $(\sim 60 \mathrm{pc})$ linear radio structure oriented roughly north-south (Neff \& de Bruyn 1983; Ulvestad et al. 1999; Antón et al. 2002). Baum et al. (1993) observed large-scale radio lobes $(\sim 6 \mathrm{kpc}$ extent) that roughly align with the small-scale jet structure. Using 5.0 GHz VLA "D" array observations, Gallimore et al. (2006) report the presence of extended emission such that the large-scale lobes are marginally resolved after the nuclear point source subtraction. In our GMRT observations, the radio emission $610 \mathrm{MHz}$ and $240 \mathrm{MHz}$ is seen as nearly point source, which we fit with single Gaussian components of sizes $\sim 6$ '. $34 \times 5$ ". 81 and $\sim 6$ '. $34 \times 55^{\prime \prime} 81$, respectively. The radio spectrum of MRK 348 fitted over $240 \mathrm{MHz}$ to $1.4 \mathrm{GHz}$ has in$\operatorname{dex}\left(\alpha_{\text {int }}\right) \sim-0.58$. The $5.0 \mathrm{GHz}$ flux density appears to be dominated by variable AGN core component and does not fit with the spectral index measured over $240 \mathrm{MHz}$ to $1.4 \mathrm{GHz}$.

\section{A.12. MRK 1}

VLA observations of MRK 1 show an unresolved point source radio emission from AGN (Kinney et al. 2000). In our GMRT observations, $610 \mathrm{MHz}$ and $240 \mathrm{MHz}$ radio images are like point-source emission and are fitted with single Gaussian components of sizes $\sim 6^{\prime \prime} .31 \times 5^{\prime \prime} .32$ and $\sim 17^{\prime \prime} .57 \times 12^{\prime \prime} .36$, respectively. The four-point radio spectrum of MRK 1 is fitted with an index $\left(\alpha_{\text {int }}\right) \sim-0.58$.

\section{A.13. MRK 1066}

The 4.9 GHz VLA observations of MRK 1066 show a linear, probably triple, source extending $\sim 2$ '. 8 along PA $\sim 134^{\circ}$ (Ulvestad \& Wilson 1989). Using 1.4 GHz and 8.4 GHz VLA observations Nagar et al. (1999) reported that the $1.4 \mathrm{GHz}$ radio emission can be fitted with a single Gaussian component and $8.4 \mathrm{GHz}$ radio emission displays the central source and northwest extension along the position angle $\sim 305^{\circ}$ with respect to the core. A more diffuse southeast extension along the position angle $\sim 140^{\circ}$ with respect to the core also seems to be present (Nagar et al. 1999). In our GMRT observations, $610 \mathrm{MHz}$ and $240 \mathrm{MHz}$ radio images show nearly point-source emission and are fitted with single Gaussian components of sizes $\sim 66^{\prime \prime} 67 \times 5^{\prime \prime}$. 63 and $\sim 17^{\prime \prime}$. $25 \times 13^{\prime \prime}$. 53 , respectively. The integrated radio spectral index fitted over $240 \mathrm{MHz}$ to $5.0 \mathrm{GHz}$ is $\sim-0.72$.

\section{A.14. NGC 2110}

VLA observations show a symmetrical, jet-like radio emission, extending $\sim 4^{\prime \prime}$ in the north-south direction and straddling a central compact core (Ulvestad \& Wilson 1984b). Using $1.4 \mathrm{GHz}$ and $8.4 \mathrm{GHz}$ VLA (A and AnB hybrid array configuration) observations, Nagar et al. (1999) reported $1.4 \mathrm{GHz}$ emission as a point-source emission, while at $8.4 \mathrm{GHz}$ a linear jet-like structure was noticed along the position angle $\sim 9^{\circ}$. In our GMRT observations, the radio emission at $610 \mathrm{MHz}$ and $240 \mathrm{MHz}$ are are seen as nearly point-source emission. We fit $610 \mathrm{MHz}$ and $240 \mathrm{MHz}$ emission with single Gaussian components of sizes $\sim 7$ ". $68 \times 5$ ". 11 and $\sim 38$ '. $^{\prime} 85 \times 32$ '. 47 , respectively. The four-point radio spectrum of NGC 2110 is steep with an integrated spectral index $\left(\alpha_{\text {int }}\right) \sim-0.72$.

\section{A.15. NGC 2273}

The 5.0 GHz sub-arcsec resolution VLA observations of NGC 2273 show an unequal double with a separation of $\sim 1^{\prime \prime}$. 2 $(\sim 145 \mathrm{pc})$ at $\mathrm{PA} 90^{\circ}$. While observations at $1.4 \mathrm{GHz}$ show the double structure embedded in a more amorphous structure with an extent of $2^{\prime \prime}$.5 ( $\sim 300 \mathrm{pc}$ ) along PA $20^{\circ}$ (Ulvestad \& Wilson 1984b). The 5.0 GHz WSRT observations show an additional amorphous structure on a larger scale and suggest an extended emission with a total extent of $\sim 9^{\prime \prime}(\sim 1 \mathrm{kpc})$ along PA $160^{\circ}$ (Baum et al. 1993). In our GMRT observations, the radio images at $610 \mathrm{MHz}$ and $240 \mathrm{MHz}$ show nearly point source emission that is fitted with single Gaussian components of $\sim 8^{\prime \prime} .32 \times 5$ ". 34 and $\sim 20$. $84 \times 11^{\prime \prime}$. 53 , respectively. The $610 \mathrm{MHz}$ radio image appears to be marginally resolved. The four-point radio spectrum of NGC 2273 is relatively flat with an integrated spectral index $\left(\alpha_{\text {int }}\right) \sim-0.37$. 


\section{A.16. NGC 5252}

The $1.4 \mathrm{GHz}$ and $5 \mathrm{GHz}$ VLA observations show a radio structure consisting of a central, compact core, with a relatively flat spectrum and a weaker emission extending $\sim 2^{\prime \prime}$ north and south of the core (Wilson \& Tsvetanov 1994). Nagar et al. (1999) confirm the overall flatter spectrum of the core $\left(\alpha_{5.0 \mathrm{GHz}}^{1.4 \mathrm{GHz}} \sim-0.32\right)$ and the radio continuum features seen in the earlier observations. In our GMRT observations, we fit the radio emission at $610 \mathrm{MHz}$ and $240 \mathrm{MHz}$ with single Gaussian components of sizes $\sim 10$ '. $56 \times 55^{\prime \prime} .66$ and $\sim 466^{\prime \prime} 20 \times 19^{\prime \prime} 31$, respectively. The four-point radio spectrum of NGC 5252 is flat with integrated spectral index $\left(\alpha_{\text {int }}\right) \sim-0.14$, suggesting that the radio emission over $240 \mathrm{MHz}$ to $5.0 \mathrm{GHz}$ is dominated by a compact AGN core.

\section{A.17. NGC 5728}

The $5.0 \mathrm{GHz}$ and $14.9 \mathrm{GHz}$ VLA observations of NGC 5728 show a compact core and a faint radial feature extending along north-east (Schommer et al. 1988). In our $610 \mathrm{MHz}$ GMRT image we noticed a double-peaked emission where the second off-nuclear component may correspond to the nuclear starforming region reported in previous studies (Schommer et al. 1988; Mazzuca et al. 2008). The peaks of the two emitting components are separated by $\sim 10 ! 7(\sim 2.0 \mathrm{kpc})$ with the offnuclear component residing along PA $\sim 67^{\circ}$ from the central nuclear component. The two components have peak flux densities of $\sim 25.6 \mathrm{mJy} /$ beam and $17.5 \mathrm{mJy} /$ beam and are fitted with Gaussians of $\sim 12$ '. $60 \times 77^{\prime \prime} 46$ and $\sim 16$ '. $10 \times 9$ !' 39 , respectively. The $240 \mathrm{MHz}$ radio image shows one component of emission (possibly due larger synthesized beam-size) fitted with a Gaussian of $\sim 37$ '. $62 \times 19$ '.20. The four-point radio spectrum exhibits inverted shape with turnover between $610 \mathrm{MHz}$ and $1.4 \mathrm{GHz}$.

\section{A.18. NGC 7212}

VLA observations of NGC 7212 show a compact double source separated by $\sim 0$.'7 in the north-south direction, and the northern blob appears to be slightly elongated (Falcke et al. 1998). In our GMRT observations, the $610 \mathrm{MHz}$ image shows two emission components. The radio contours overlaid on DSS optical image clearly show that the second radio component is associated with the neighboring galaxy NGC 7213. The radio emission component associated with NGC 7212 is fitted with a single
Gaussian of $\sim 8$ ". $40 \times 4$ "' 85 . Owing the large synthesized beam size (i.e., $\sim 40$ ' $16 \times 19$ ' 61 ), the $240 \mathrm{MHz}$ radio image shows only one emission component that includes emission from NGC 7213 too. The $240 \mathrm{MHz}$ radio emission is fitted with a Gaussian of $\sim 32^{\prime \prime}$. $85 \times 25^{\prime \prime}$. 79 . The large beam size at $240 \mathrm{MHz}$ does not allow us to estimate the flux densities of NGC 7212 and NGC 7213 separately. Therefore, $240 \mathrm{MHz}$ flux density measurement of NGC 7212 is contaminated and over-estimated. The four-point integrated radio spectral index of NGC 7212 is $\sim-0.79$. We note that spectral steepening seen over $610 \mathrm{MHz}$ to $240 \mathrm{MHz}$ is likely to be caused by the over-estimated $240 \mathrm{MHz}$ flux density. It can also give rise to the steeper value of the integrated spectral index than the actual value.

\section{A.19. NGC 7682}

The $8.4 \mathrm{GHz}$ VLA observations of NGC 7682 show unresolved point-source nuclear emission (Kukula et al. 1995); however, the milli-arcsec 5.0 GHz VLBI observations show a point source with extension along the southern (PA $\sim 180^{\circ}$ ) and the southeastern $\left(\mathrm{PA} \sim 120^{\circ}\right.$ ) directions (Lal et al. 2004). Our GMRT images at $610 \mathrm{MHz}$ and $240 \mathrm{MHz}$ are fitted with single Gaussian components of sizes $\sim 10$.' $21 \times 66^{\prime \prime} 82$ and $\sim 38^{\prime \prime} .68 \times 29$ '. 23 , respectively. The four-point spectrum of NGC 7682 is steep with spectral index $\left(\alpha_{\text {int }}\right) \sim-0.72$.

\section{A.20. MRK 533}

VLA "C" array observations of MRK 533 at $8.4 \mathrm{GHz}$ show a slightly extended nuclear emission, which is seen as resolved into a 0.5 arcsec double structure in VLA "A" array observations (Kukula et al. 1995). VLA and EVN observations reveal a linear triple radio source of $\sim 0$.' 7 angular extent with the components at $\sim 0$.' 5 west and $\sim 0$.' 15 east of the main peak (Unger et al. 1988). Momjian et al. (2003) made more sensitive observations of MRK 533 using VLBA, phased VLA and Arecibo at $1.4 \mathrm{GHz}$ and report the triple source and additional lowsurface-brightness emission forming an S-shaped structure. Our GMRT images at $610 \mathrm{MHz}$ and $240 \mathrm{MHz}$ show nearly pointsource radio emission that is fitted with single Gaussian components of sizes $\sim 66^{\prime \prime} 98 \times 6$ '. 26 and $\sim 20$.' $^{\prime} 01 \times 19$.' 04 , respectively. The radio spectrum of MRK 533 over $240 \mathrm{MHz}$ to $5.0 \mathrm{MHz}$ is fairly steep with spectral index $\left(\alpha_{\text {int }}\right) \sim-0.78$. 